\title{
Cooperative Social Robots to Accompany Groups of People
}

\author{
Anais Garrell \\ Mobile Robotics Group \\ Institut de Robòtica i Informàtica Industrial CSIC-UPC \\ Barcelona-Spain \\ agarrell@iri.upc.edu \\ Alberto Sanfeliu \\ Mobile Robotics Group \\ Institut de Robòtica i Informàtica Industrial CSIC-UPC \\ Barcelona-Spain \\ sanfeliu@iri.upc.edu \\ *
}

\begin{abstract}
This study proposes a new model for guiding people in urban settings using multiple robots that work cooperatively. More specifically, this investigation describes the circumstances in which people might stray from the formation when following different robots' instructions. To this end, we introduce a "Prediction and Anticipation Model" that predicts the position of the group using a Particle Filter, while determining the optimal robot behavior to help people stay in the group in areas where they may become distracted. As a result, this article presents a novel approach to locally optimizing the work performed by robots and people using the minimum robots' work criterion and determining human-friendly types of movements. The guidance missions were carried out in urban areas that included multiple conflict areas and obstacles. This study also provides an analysis of robots' behavioral reactions to people by simulating different situations in the locations that were used for the investigation. The method was tested through simulations that took into account the difficulties and technological constraints derived from real-life situations. Despites these problematic issues, we were able to demonstrate the robots' effect on people in real-life situations in terms of pushing and dragging forces.
\end{abstract}

*This research was conducted at the Institut de Robòtica i Informàtica Industrial (CSICUPC). It was partially supported by the CICYT project RobTaskCoop (DPI2010-17112) and the MIPRCV Ingenio Consolider 2010 (CSD2007-018). 


\section{Introduction}

In recent years, research and wide-spread interest in the interaction between robots and humans have increased rapidly, in the academic sphere as well as in laboratories, private companies, and the media. The applications of this field are very diverse, ranging from developing automatic exploration sites (Trevai et al., 2003) to using robot formations to transport and evacuate people in emergency situations (Casper and Murphy, 2003). Researchers are also hard at work on crafting robots that can operate as team members (Scholtz, 2002), therapists (Dautenhahn, 1999) and in robotics services (Kawamura et al., 1996).

Within the area of social and cooperative robots, the nature of interactions between a group of people and a set of accompanying robots has become a primary point of interest.

Our findings are based on the behavior of a team of robots which operate cooperatively to accompany a group of people from a designated starting point to a specific destination within an urban area. One of the robots is the leader, it works as a human tour-guide. It is placed at the front of the formation. The other robots, known as shepherds, are responsible for helping people to stay in the group, and to make sure they follow the path indicated by the leader. This study suggests that robots can be aware of people's positions. Our research focuses specifically on the behavior of robots accompanying groups of people in different urban environments.

In previous studies (Garrell and Sanfeliu, 2010b), we introduced a representative model of the environment in which robots were able to perform their assigned tasks. This investigation takes this one step further by presenting a "Prediction and Anticipation Model" (PAM). This model projects the behavior of a group of people (prediction) using a particle filter (Arulampalam et al., 2002). It also enables us to determine the particular distribution of robots that can be used to best prevent people from straying from the formation in specific areas of a map (anticipation). By using the Prediction and Anticipation Model, we were able to prevent people from straying from the guided group and, thus facilitate the task of the robots. Furthermore, we found we could locally optimize the work performed by robots and people alike, and therefore obtain a human-friendly motion.

To test the proper functioning of the model, we conducted several simulations. We were not able to test the complete method in real-life scenarios due to technological constraints and safety concerns (due to a lack of robots equipped with high acceleration capabilities and that were also deemed safe to navigate in urban sites). Despite these factors, we were able to successfully evaluate the effect of a robot's pushing and dragging forces on people in real-life situations using one of our robots (Tibi).

In the simulations, a group of people were led by a group of robots in an urban area that involved navigation through and around several cross-streets, buildings and obstacles. The simulations that were performed encompassed a variety of different factors including: (i) number of people being accompanied, (ii) different layout scenarios within the environment (for example, corridors, 


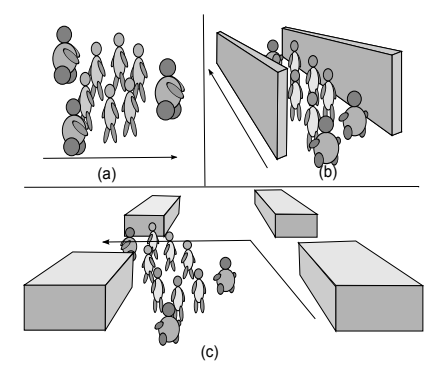

Figure 1: Different areas in the environment where robots performed their guiding task. (a) open areas, (b) narrow corridors, and (c) intersections.

open areas or intersections), and (iii) situations in which a specific number of people strayed from the designated path at the same time. In the simulations, we compared two alternative strategies: (i) placing a robot of the initial formation in the conflict area, in order to prevent people from straying, and inviting people to return to the formation using voice instructions via the PAM model. (ii) Wherever participants strayed, we studied the robots' behavior and evaluated how they were able to solve this kind of herding task without necessarily having anticipated the situation. In each of these two strategies, we measured the time it took to perform the task and assessed the robots' performance in order to determine the best strategy for each situation.

We also conducted real-life experiments to analyze the robots' impact on people when they are guided by the pushing and dragging forces that exist between robots and humans, or even between humans and humans. These forces have been identified and quantified in prior studies of pedestrians in crowds and in evacuation scenarios (Helbing and P., 1997, Shell and Matari, 2005). To ensure that the above mentioned model was valid for our study, we conducted a series of real-life experiments that include either: a robot approaching a moving person from different angles at speeds, or a person following a robot. We compared the results of these tests with those yielded by the simulations, in order to demonstrate the proper functionality of the model.

In the remainder of the paper we start by introducing the Related Work of social and companion robots. Section 3 provides an overview of the method we describe in this article, including problems constraints and assumptions. Section 4 introduces the model of people's movements. Section 5 describes the prediction and anticipation model and outlines the methods for identifying high risk areas on a map. In section 6 , the definition of the optimal robot task assignment for a cooperative mission is presented. The computation of robot configurations for group reunification is shown in Section 7 . The results, a discussion of the methods used and conclusions are presented in Sections 8, 9 and 10 respectively. This paper is an expanded version of the work presented in (Garrell and Sanfeliu, 2010a). In this current version we have included a new model called Prediction and Anticipation Model which has been developed to predict human behavior and prevent people from straying from the formation. 


\section{Related Work}

The evolution of robotics and the creation of robots with a greater capacity to interact with human beings, have together led to the development of cognitive robots equipped to assist people by acting as assistants and companions. However, for robots to truly be accepted as companions, they need to have communicative capabilities similar to those of humans. An autonomous companion robot should be seen as a special type of service robot, specifically designed for personal home use. Companion robots are expected to be able to communicate with non-expert users in a natural and intuitive way.

Research into human-robot interaction in the field of companion robots is still relatively new in comparison to traditional service robotics, such as robots serving food in hospitals or providing specific security services. Therefore, prior research in this particular field is relatively minimal (Wilkes et al., 1997). Most of the current research predominantly studies robots that participate in socialhuman interactions as companions (Ishiguro et al., 2001). Researchers are currently working on developing this type of robot (Hornby et al., 2000). Many studies have investigated people's attitudes towards robots and their perception of robots. For example, robotic dogs such as AIBO, developed by Sony (Kaplan, 2000), are autonomous robots created for entertainment purposes; they are made for home use and are capable of provoking emotions, showing instinct, learning and developing their skills, as well as interacting with children. The design for AIBO was based on a dog's appearance and behavior (Melson et al., 2005 ) and various studies have proven that AIBO is capable of forming psychological relationships with adults and children alike (Kubinyi et al., 2004).

The work of Pransky (Pransky, 2004) brought a new perspective to the different uses and identities of a companion robot for the future by describing the advantages and disadvantages of this type of companion. The "Robotic Butler/Maid" was able to perform domestic tasks, but also caused difficulties in relationships at home by being too efficient and making people feel redundant. The "Robot Nanny" could play with children and feed them, but at times also prevented them from having meaningful interactions with humans and seeing interactions as normal. Or there may be a case where a companion robot, that acted as an assistant, would be able to organize meetings and correct documents but might give one the feeling that this interaction with a robot is easier than engaging in any human contact at all.

In (Dautenhahn et al., 2005), a human-centered approach was adopted in order to look into people's perceptions and their desires for a companion robot. If social robots are going to be used in office and domestic environments, where they will have to interact with different individuals, they will have to be able to survive and perform tasks in dynamic, unpredictable environments and they must act safely and efficiently. The presence of human beings creates new problems for motion planning and control, as their security and comfort must be considered. The principal goal of the motion planner is to take human movements into account in order to ensure their safety. This requires both safe and socially acceptable routes to be determined. 
Within the field of companion robots, a small number of studies describe the behavior of robots when guiding a person or group of people.

Vaughan introduced a complete robot system which controlled the behavior of another intelligent system with the presence of variability, uncertainty and noise (Vaughan et al., 2000). The robots used in the Sheepdog Project demonstrated the ability to gather a flock of ducks and carry out maneuvers to safely deliver them to a predetermined point. The use of ducks instead of sheep made it possible to conduct the experiment in a controlled environment. More importantly, the behavior of flocks of ducks is considered by shepherds to be similar to that of sheep; in fact, ducks are sometimes used to train sheepdogs due to their relatively slow movements. A generalized model of group behavior was designed in order to identify animal-robot interaction. The hypothesis posited that if the model accurately captures the basis of behavior, then the system controlling the model should be able to control behavior in the real world.

Later in the study, it was suggested that it would be possible to describe this behavior according yo a model of the force of attraction between animals, with the magnitude of attraction varying with the inverse square of the mutual distance between the animals (Harrison, 1999). The relationship was purported to represent a linear response in sensorial information, which also varies with the inverse square of distance. Other models have yielded similar results with flocks of birds (Vaughan, 1998).

These ideas have been adapted to robotics where these techniques are used from potential fields for navigation (Marques and Lima, 2002). This type of algorithm uses the analogy of forces which act on particles, such that the robot moves as though it were a particle, attracted or repelled alternatively by the characteristics of its surroundings. A robot is usually attracted to an object and repelled by obstacles.

Once the social behavior of animals was analyzed, the next step was to study how people react to the robot's reactions. Since the beginning of biologically inspired robots, researchers have been fascinated by the possibilities of interaction between robots and their surroundings, and the possibility of their ability to interact amongst themselves. These groups of social robots are designed to maximize benefits (for example, performing tasks) through collective action. Until now, research has used the principles of self-organization and social behavior based on interactions within groups of hymenopterous insects, anonymous, homogeneous groups where unique elements are insignificant. This type of social behavior has proved to be an attractive model for robotics, especially since it allows groups of relatively simple robots to perform difficult tasks.

The development of a robot as a "social individual" requires the use of different models and techniques based on "social groups" for robots that are working collectively. In particular, learning and social imitation, gestures and natural language for communication, as well as emotion and recognition of interaction by all partners are all important factors. Furthermore, most research in this field has focused on the application of social behaviors and, as a result, social robots are generally undertood to be assistants, companions or pets.

Studies performed on robots, most of which serve to guide humans have been 
conducted in museums (Burgard et al., 1998, Nourbakhsh et al., 2003, Davies et al., 1998), railway stations (Hayashi et al., 2007), hospitals (Feil-Seifer and Mataric, 2005), as assistants (Dautenhahn et al., 2005, Sabanovic et al., 2006), and in shopping malls (Shiomi et al., 2009); other studies have examined ways in which robots provide humans with the flexibility of deciding the way in which they want to be guided (Pandey and Alami, 2009). In all these scenarios, the primary purpose of the robots was simply to educate or entertain people.

However, various new projects in which robots accompany humans have recently demonstrated robots can not only determine safe paths, but also plan out socially acceptable routes for humans (Sisbot et al., 2005). Robots operating around people should move coherently and in easily-understood ways, so they do not endanger the humans in their company. As a result, they could show that they are capable of acquiring spatial social skills (Hayashi et al., 2007).

Some researchers have developed projects which introduce group attention control, a system which allows robots to interact with a group of people (Shiomi et al., 2007). (Shiomi et al., 2010) looks into how human-robot interaction changes when a robot moves forward or backward as people move around it.

The above mentioned methods consider either single robots or multiple robots moving independently. To our knowledge, only a small handful of studies discuss multiple robots behaving in cooperative modes. For instance, (Martinez Garcia et al., 2005) performed a qualitative analysis of the movements of different entities, humans or animals, and built a multi-robot sytem architecture of three robots to guide them. However, they failed to consider realistic situations which involve obstacles or people straying from the group. In a study carried out by Casper and Murphy, they tested several types of robot formations and different strategies for drawing the robots to people (Casper and Murphy, 2003). However, these issues and the general movements of the robots are governed by a large number of heuristics, which makes the system impractical.

Furthermore, in (Garrell et al., 2009) a model capable of representing the whole environment in which robots perform guidance tasks within an open and unbounded area with obstacles was employed. Their model shows how the elements of this environment relate to the group of robots and people. It is also able to estimate the position, orientation and velocity of the robots and people, as well as the position of the obstacles at a certain point in time. It is also capable of predicting the intersection of the people with the obstacles and detecting if someone is leaving the group using a Particle Filter (Arulampalam et al., 2002). It computes the robots' trajectory to achieve this goal while preventing people from leaving the group.

In contrast to the previous approaches, the "Prediction and Anticipation Model" proposed offers a new framework for tackling more realistic situations, without needing to use such a large number of heuristics, by allowing robots to prevent people from straying from the formation, which is undeniably among the most challenging aspects of this topic. 


\section{Overview of the Method}

As stated earlier, this article describes a new model that has been designed to accompany people using a team of robots which work cooperatively to perform the assigned task. One of the main issues which arises when humans are asked to follow robots' instructions in a given task is the fact that people might stray from the robot formation. On the one hand, the main focus of this study is on the "Prediction and Anticipation Model" (PAM) of human behavioral motion. We introduce a model which suggests a probable position of the members of the group using a Particle Filter (prediction), and it attempts to determine the optimal number of robots needed using the minimum robot energy (or work) criterion, depending on the number of individuals present and the environment in which they find themselves. We then go on to analyze the areas of the established map which could cause confusion in the group or result in general group dispersion, and explore ways of preventing people from straying from the path by considering the optimal formation of the robots in terms of the task that needs to be performed (anticipation).

On the other hand, although robots might be able to anticipate human actions, people might also move away from the group spontaneously. In light of this, our article introduces a novel approach to locally optimize the work performed by robots and obtain a human-friendly motion (basically, ensuring that robots do not intrude on the humans' personal space). We consider situations in which individuals can move away from the formation, or in which they must be regrouped by multiple mobile robots working cooperatively. These issues are addressed by introducing a new cost function which minimizes the work required by robots to lead and regroup people.

\subsection{Problem Constraints and Model Assumptions}

This subsection describes the limitations we came across while testing our model in real-life scenarios and which led us to continue testing exclusively through simulations. We will also explain the model assumptions we adopted for the simulations.

There are at least two types of problem constraints. The first derives from robots' perception systems and motions; the second one is the result of human behavioral reactions to the robots' instructions. The effects of these limitations on our study are summarized below:

- Robots cannot accelerate or go faster than people in a safe way. This means our robots cannot usually follow people's behavioral reactions.

- The localization of urban robots in dense environments has not been tested widely, and existing methods are not yet robust enough.

- No robust methods exist to detect people's poses (position and orientation) when they move in a group. From the point of view of a robot on one of the sides of the group, some individuals are inevitably partially or completely 
out of sight. If the group has a small number of people the chances of achieving accurate pose detection are high, but if there are many people, detection becomes very difficult. Since our method is based on estimating the pose of each person of the group, pose detection is key to our research.

- There is not currently enough data on people's reactions to robots' motion instructions. Instructions like "please return to the group" or "do not go away" might cause different reactive motions in different touring situations.

Because of these limitations, and due to the lack of knowledge of other reallife experimental works, we have made the following assumptions in our model:

- People in a guided tour "usually" follow the group leader. In this case, the leader is a robot.

- People understand, agree with and follow the robot's instructions and motions (for example, when a robot approaches a person who has moved away, the person follows the robot's instructions and movements).

- The number of people who move away from the formation is no more that one or two people.

- When one or more robots are used to block one area to form a barrier people do not attempt to cross the robot barrier.

- After 15 minutes of a robot guidance, $26 \%$ of people stray from the group, see (Nourbakhsh et al., 2003) for additional information.

- Robots know the pose (position and orientation) of each person in the group.

- Robots are always localized keeping in mind the constraints of the physical environment.

- Robots can communicate between them and send and receive information.

- Robots have a map of the environment in which the tour or visit is going to take place.

- Robots have enough acceleration capacity and speed to safely reach a person, who has strayed.

The first four assumptions are usually demonstrated by people who follow the "citizen rules" of common civic behavior and who are not in a state of panic or engaging in an act of vandalism. As a result, we are assuming people understand and obey the tour guides. The fifth assumption has been observed in social studies carried out on human motion behavior (Nourbakhsh et al., 2003). The sixth assumption is difficult to verify at the present time because, although there exist several algorithms which can detect people's position, the 
methods are not yet precise and robust enough for situations in which people are partially or completely out of the robots's field of vision, for example (Konigs and Schulz, 2011). The next three assumptions are valid and have been tested in outdoor real-life scenarios (Murtra et al., 2008). The last assumption is also complicated and, although there are many good and fast robots with excellent navigation methods (Trulls et al., 2011), there are no safe robust methods that have been tested in real-life experiments with people. Moreover, robots currently do not have the adequate acceleration and prediction methods to follow people's random and spontaneous changes of motion.

\subsection{Paper Topics and Contributions}

The contributions and topics described in the paper are presented below, see Fig. 2.

- Representation of people's motion: Before working with real robots and real people, we first must develop some simulation results. In order to do this, we needed to model people's motion by using the concepts introduced in the investigation carried out by Helbing et al., which studied the dynamics of pedestrian crowds from the "social force model" point of view (Helbing and Molnar, 1995). The meaning of "social force model" does not refer to a social robot's behavior, but rather to the existence of a non-physical force (push, drag or traverse) robots can exert to move or drag people. More specifically, this work describes the pedestrians' motion based on a social force model which result from the internal motivations of the individuals performing certain movements (Zanlungo et al., 2011).

- Prevent group dispersion: As indicated earlier, our work focuses on the behavior of robots when guiding a group of people in urban areas. One of the main challenges when robots try to perform this task is the possibility that one or more people will move away from the group, either out of boredom or due to his/her interest in something which lies away from the group's path.

To use the model presented in this work, Prediction and Anticipation Model (PAM), we needed to know the layout of the urban map, the path that would be taken, the final destination of the guided group, and the estimated positions of the people and robots. The first component of the model is the prediction, which anticipates the movements that will be made by both the humans and robots. The second component, anticipation, detects the conflict areas (where people are prone to stray) and calculates the number of robots that should cover the conflict area. The Prediction and Anticipation Model (PAM) uses the previously gathered data (i.e. people's and robots' positions, detection of conflict areas, etc.) to compute the possible distribution of robots in order to cover the conflict areas and guide the people to the final destination. Because we needed to minimize the number of robots and wasted energy, the PAM is designed 
to seek out the number of robots and determine their respective tasks so as to minimize the work done by robots and people.

- Detection of conflict areas: As previously indicated, one of the key components of our work is the robots' ability to anticipate human movements. Therefore, it is crucial to understand the environment in which the group moves. As a result, we needed to know the set of robots and people that would be situated in the hallways, open spaces, intersections and other settings. We also describe a function which determines the density of obstacles that surround the group during their movement along the path. In the areas with open spaces and a low density of obstacles, the probability that a person will move away is high and, thus, these areas were treated as conflict areas in our investigation.

- Minimum work performed by robots and people: We present a function which determines the optimal configuration of robots (defined as the behavior of all the members of the group). That configuration should not only minimize the work performed by the robots, but also make the interaction between robots and people as "comfortable" as possible, in terms of motion (in this context, the word "comfortable" denotes that the robots will not try to intrude on people's personal space). This function can be used in two different stages of the simulations: (i) once we have determined that areas in which people are more likely stray, we can asses which robot ought to be sent to the area; and, (ii) when that person leaves the group, we can also calculate which robot should be sent to find and accompany him/her back to the formation. In both cases, we determined the strategy for assigning robots' tasks which required the least amount of work and also led to the minimum displacement problems in the guidance task. Moreover, robots had to be able to perform all their tasks while navigating quietly, avoiding obstacles, and not intruding on people's personal space.

- Regrouping people: In our work, we allowed for the possibility that one or more people would move away from the group. The system would then need to assign a robot to perform the task of retrieving him/her. By using the function introduced above, we computed the behavior of the team of robots to redirect the person who has strayed. Once the configuration with minimal work cost was determined, we could compute the robot's trajectory to regroup the people.

\section{People Motion}

The model presented in this article was developed to be applied to robots. But, in order to conduct simulations and test the robots' efficiency and correct behavior, we relied on models of both robots' and people's movements. To model 


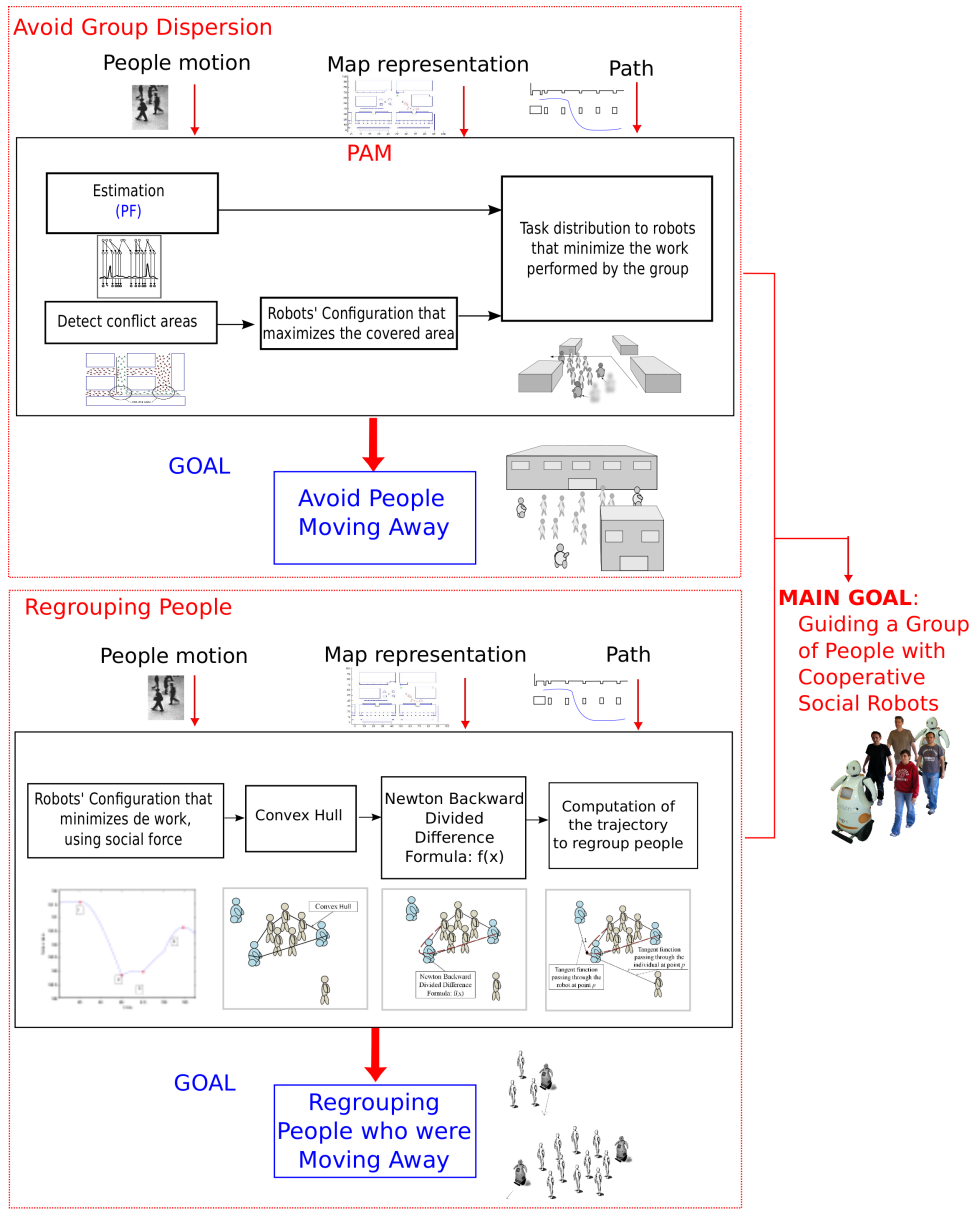

Figure 2: Overview of the study presented. An essential part of our work was to simulate people's movements and to show humans' reactions depending on the stimulus provoked on them. We also offer solutions to two different problems that were found while carrying out the mission of guiding people. First, we show the PAM model, to prevent group dispersion, we identify areas where people might get lost, and robots are located there to bring them back to the formation. Secondly, if someone moves away from the group, the behavior of the robots to accompany him/her back to the formation is shown. For both problems, we use a function that minimizes the cost of work of robots and humans in order to make the interaction between them as pleasant as possible.

people's behavior, we used the concepts introduced by Helbing et al. (Helbing and Molnar, 1995). At first, one might think that these forces are chaotic and therefore unpredictable. However, in some simple situations, they can be described through probabilistic models. The situations treated in previous approaches are: (i) When the pedestrian wants to reach a certain destination 
with the minimum robot disturbance. (ii) When the motion of a pedestrian is influenced not only by other pedestrians but also, in our case, by the robots. And, (iii) The fact that pedestrians are sometimes attracted by other people or objects outside the bounds of the group.

Since the third approach corresponds essentially to a situation in which a person strays from the group and, as this instance would be controlled by the robots, we only took into account the first two situations. Situations (i) and (ii) can be represented mathematically.

For the first situation, people usually take the shortest path, which might be formally represented as the shape of a polygon with edges $\vec{r}_{\alpha}^{1} \ldots \vec{r}_{\alpha}^{n}:=\vec{r}_{\alpha}^{0}$, where $\alpha$ refers to a given person and $\vec{r}_{\alpha}^{0}$ the destination he/she wants to reach.

The desired motion direction $\vec{e}_{\alpha}(t)$ of a pedestrian $\alpha$ will then be:

$$
\vec{e}_{\alpha}(t):=\frac{\vec{r}_{\alpha}^{k}-\vec{r}_{\alpha}(t)}{\left\|\vec{r}_{\alpha}^{k}-\vec{r}_{\alpha}(t)\right\|}
$$

where $\overrightarrow{r_{\alpha}}(t)$ is the current position and $\vec{r}_{\alpha}^{k}$ is the subsequent edge of the polygon that will be reached. A deviation of the desired speed, $v_{\alpha}$, from the current velocity, $\vec{v}_{\alpha}(t):=v_{\alpha} \vec{e}_{\alpha}(t)$, may also exist due to deceleration or obstacle avoidance processes:

$$
\vec{F}_{\alpha}^{0}\left(\vec{v}_{\alpha}, v_{\alpha} \vec{e}_{\alpha}\right):=\frac{1}{\tau_{\alpha}}\left(v_{\alpha} \vec{e}_{\alpha}-\vec{v}_{\alpha}\right)
$$

where $\tau_{\alpha}$ is a relaxation term. In practice we set the term $\tau$ to 0.5 for all pedestrians.

Secondly, let us now consider situation (ii), when a pedestrian's motion is influenced by other people from the group. This situation is related to the fact that each individual in the group tries to maintain an empty safety space around themselves (Worchel et al., 2002). This is, in fact, a repulsive effect which we model through the following vectorial quantity:

$$
\vec{F}_{\alpha \beta}\left(\vec{r}_{\alpha \beta}\right)=-\nabla_{\vec{r}_{\alpha \beta}} V_{\alpha \beta}\left[b\left(\vec{r}_{\alpha \beta}\right)\right]
$$

where $V_{\alpha \beta}(b)$ is a repulsive potential which is assumed to be a monotonic decreasing function of $b$ with equipotential lines having an elliptical shape. $\vec{r}_{\alpha \beta}=\vec{r}_{\alpha}-\vec{r}_{\beta}$. The parameter $b$ denotes the semi-minor axis of the ellipse and is given by:

$$
b=\frac{\sqrt{\left(\left\|\vec{r}_{\alpha \beta}\right\|+\left\|\vec{r}_{\alpha \beta}-v_{\beta} \Delta t \vec{e}_{\beta}\right\|\right)^{2}-\left(v_{\beta} \Delta t\right)^{2}}}{2}
$$

where, $v_{\beta} \Delta t$ is an approximation the size of a step taken by a pedestrian $\beta$.

Finally, we consider the repulsive effect produced by obstacles that are in the environment where as a result of such force, people try to keep a distance from those obstacles. The nature of this force is the same as the one we just described between individuals with the difference being that the obstacles do not move. If we denote $B$ as the border of an obstacle, then the repulsive effect created by it will be described by: 


$$
\vec{F}_{\alpha B}\left(\vec{r}_{\alpha B}\right)=-\nabla_{\vec{r}_{\alpha B}} U_{\alpha B}\left(\left\|\vec{r}_{\alpha B}\right\|\right)
$$

where $U_{\alpha B}\left(\left\|\vec{r}_{\alpha B}\right\|\right)$ is the repulsive decreasing potential function, $\vec{r}_{\alpha B}=\vec{r}_{\alpha}-\vec{r}_{B}$, and $B$ denotes the position of the border $B$ which is closest to the pedestrian $\alpha$.

\section{$5 \quad$ Prediction and Anticipation Model}

One of the main issue when robots try to perform the task of guiding a group of people, is the possibility that one or more people move away from the group, either out of boredom or due to his/her interest in something lying outside the given path.

In a previous work (Garrell and Sanfeliu, 2010a), we studied how robots should behave when they accompany groups of people, or which strategy the group of robots should follow when a person moves away. In this work, we go one step further, presenting a model designed to prevent people from moving away from the formation. Therefore, the model for this study must incorporate both information about the environment as well as people's behavior within that environment, so robots are able to anticipate the problem of losing people.

To apply the Prediction and Anticipation Model (PAM), we must know the the layout of the urban map a priori, the path to the final destination of the guided group and the estimated positions of the people and robots. The first component of the model is the Prediction, which is used to foresee the people's and robots' motion some steps ahead. The second component, Anticipation, identifies the conflict areas (where people can move away) and computes the number of robots that should cover that conflict area. The PAM then uses this data (people's and robots' positions, detection of conflict areas) to calculate the distribution of robots to cover the conflict areas and guide the people to the destination. The PAM also decides the number and distribution of robots in a way that minimizes the work done by robots and people, see Fig.3.

\subsection{Prediction}

As previously mentioned, a key aspect of a guiding mission is to prevent people from straying from the group. To achieve this aim, the model must estimate people's positions and velocities with a Particle Filter (PF), in this case a Gaussian Particle Filter (GPF).

We have modified part of the measurement update algorithm (Arulampalam et al., 2002) to prevent humans and robots from colliding with each other and with obstacles, based on the findings in earlier studies (Liu and Chen, 1998). Furthermore, we use the information on people's motions in the Particle Filter to estimate the position of the group over time. The computation to estimate people/robots' positions is sequential, it starts from the leader robot, then people of the group, and finally shepherd robots. In each step of the process, the people/robot position (personal space occupancy) is updated. In the sampling 


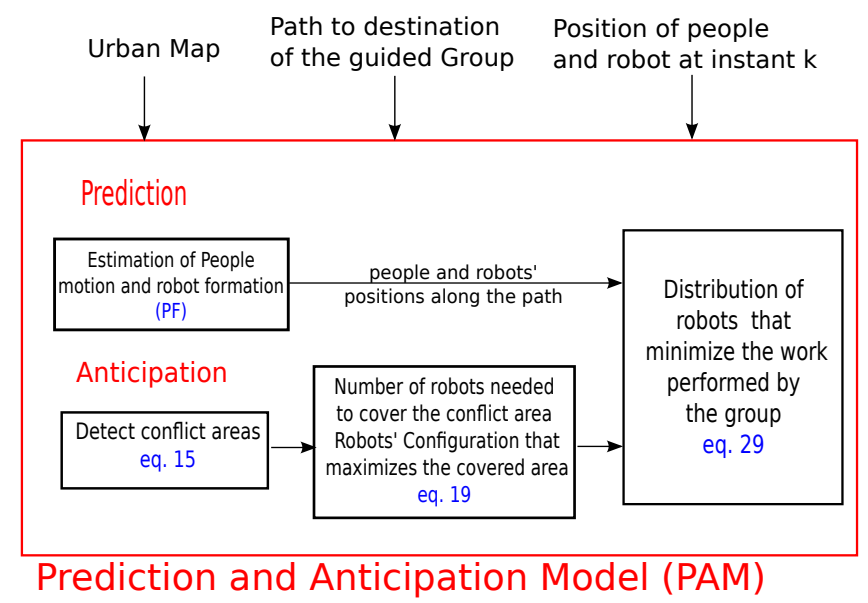

Figure 3: Components of the PAM model. The first component estimates people and robots' positions along the path by using a particle filter. The second components is anticipation, which detects the areas on the map where people might get distracted and move away, it also computes the configuration of robots which maximizes the covered area. Using the junction of the two components, the distribution of robots that minimizes the work performed by both robots and people is obtained.

process, we do not consider the particles that are located in positions already occupied by the vital space of other members of the team. So, position estimations where two different people/robots overlap the personal space occupancy of other people/robots are not allowed at any time.

The problem consists of estimating the dynamic state of a nonlinear stochastic system, based on a set of noisy observation. Our model can be written as

$$
\begin{array}{r}
x_{n}=f\left(x_{n-1}, u_{n}\right) \text { (process equation) } \\
y_{n}=h\left(x_{n}, v_{n}\right) \text { (observation equation) }
\end{array}
$$

Where $f(\cdot)$ and $h(\cdot)$ are some known functions, and $u_{n}$ and $v_{n}$ are random noise vectors of given distributions. The process equation represents a system involving time $n$, where the system is represented by the hidden state $x_{n}$. We denote by $x_{0: n}$ and $y_{0: n}$ the signal and observation up to time $n$, respectively, i.e., $x_{0: n}:=\left\{x_{0}, \ldots, x_{n}\right\}$ and $y_{0: n}:=\left\{y_{0}, \ldots, y_{n}\right\}$. Our aim is to estimate recursively in time: (i) the "filtering distribution" $P\left(x_{n} \mid y_{0: n}\right)$ at time $n$, (ii) the predictive distribution $P\left(x_{n+1} \mid y_{0: n}\right)$, both of which can be written as: 


$$
\begin{array}{r}
P\left(x_{n} \mid y_{0: n}\right)=C_{n} P\left(x_{n} \mid y_{0: n-1}\right) P\left(y_{n} \mid x_{n}\right) \\
C_{n}=\left(\int P\left(x_{n} \mid y_{0: n-1}\right) P\left(y_{n} \mid x_{n}\right) d x_{n}\right)^{-1} \\
P\left(x_{n+1} \mid y_{0: n}\right)=\int P\left(x_{n+1} \mid x_{n}\right) P\left(x_{n} \mid y_{0: n}\right) d x_{n}
\end{array}
$$

The GPF approximates the filtering and predictive distribution in eq. 8 by Gaussian densities using the particle filtering methodology. $M$ particles $X=x_{n}^{(1)}, \ldots, x_{n}^{(M)}$ from a so-called importance sampling distribution $\pi\left(x_{n}\right)$ are generated. The particles are the weighted as $w^{(j)}=\left(P\left(x_{n}^{(j)}\right)\right) /\left(\pi\left(x_{n}^{(j)}\right)\right)$. If $W=w^{(1)}, \ldots, w^{(M)}$, the set $(X, M)$ represents samples from the posterior distribution $P\left(x_{n}\right)$.

Finally, for the choice of $\pi$ we have considered:

$$
P\left(x_{n} \mid y_{0: n-1}\right)=N\left(x_{n}, \bar{\mu}_{n}, \sum_{n}^{-}\right)
$$

The change we have made in the Gaussian Particle Filter is the reweighting, which is done using a new function $g\left(x_{n}^{(j)}\right)$ (defined below in eq. 12), which sereadjusts the weights of all the set of particles $X=x_{n}^{(1)}, \ldots, x_{n}^{(M)}$. In the present work, we simulate data from different sensors to determine what the position of people who are being guided is and, therefore, it is not known with full certainty what the exact position of each person/robot is. As a result, we use a Gaussian probability distribution to determine what the most highly probable positions are. We define the position of people and robots by the Gaussian function $T_{z}\left(\mu_{z}, \Sigma_{z}\right)$

$$
T_{z}\left(\mu_{z}, \Sigma_{z}\right)(x)=\frac{1}{\left|\Sigma_{z}\right|^{1 / 2}(2 \pi)^{n / 2}} e^{-\frac{1}{2}\left(x-\mu_{z}\right)^{T} \Sigma_{z}^{-1}\left(x-\mu_{z}\right)}
$$

where $\mu_{z}=\left(\mu_{z_{x}}, \mu_{z_{y}}\right)$ is the center of gravity of the person, and $\Sigma_{z}$ is a covariance matrix whose principal axes $\left(\sigma_{x}, \sigma_{y}\right)$ represent the size of an ellipse surrounding the person (or robot) which is considered as a safety area.

We can then define people and robots by the set $\left\{\left(\mu_{x}, \mu_{y}\right),\left(\sigma_{x}, \sigma_{y}\right), v, \theta, T\right\}$, where $v$ and $\theta$ are the velocity and orientation computed by the particle filter. As we said, the variances $\left(\sigma_{x}, \sigma_{y}\right)$ represent the safety area around each person (or robot). This could be set to a constant value. However, usually one might need larger safety areas when the robots or people move faster. As a consequence, we changed the values of the variances $\sigma_{x}$ and $\sigma_{y}$ accordingly depending on the velocity parameter $v$.

For obstacles, we define their probabilities as a set of Gaussian functions placed at regular intervals around their boundaries. $X=\left\{\left(x_{1}, y_{1}\right), \ldots,\left(x_{n}, y_{n}\right)\right\}$ are denoted by the set of points evenly spaced around the boundary. Then this boundary will be defined by: $\left\{\left(x_{i}, y_{i}\right),\left(\sigma_{x_{i}}, \sigma_{y_{i}}\right), T_{i}\right\}$ for $i=1, \ldots, n$, where $T_{i}$ is described by eq. 10 . 
Therefore, once we have defined people's, robots' and obstacles's positions in a probabilistic manner, we can define the function $g\left(x_{n}^{(j)}\right)$, which is used in the measurement update algorithm (Arulampalam et al., 2002).

1. Draw samples from the function $\pi\left(x_{n} \mid y_{0: n}\right) \rightarrow\left\{x_{n}^{(j)}\right\}_{j=1}^{M}$

2. Compute the respective weights by:

$$
\begin{gathered}
\bar{w}_{n}^{(j)}=\frac{p\left(y_{n} \mid x_{n}^{(j)}\right) N\left(x_{n}=x_{n}^{(j)}, \bar{\mu}_{n}, \bar{\sum}_{n}\right)}{\pi\left(x_{n}^{(j)} \mid y_{0: n}\right)} g\left(x_{n}^{(j)}\right) \\
g\left(x_{n}^{(j)}\right)=\left\{\begin{array}{l}
\alpha \text { if } T_{\text {total }}\left(x_{n}^{(j)}\right)=0, \alpha>>1 \\
0 \text { if } T_{\text {total }}\left(x_{n}^{(j)}\right)=\infty \\
\frac{1}{T_{\text {total }}^{(j)}\left(x_{n}^{(j)}\right)} \text { otherwise }
\end{array}\right.
\end{gathered}
$$

3. Normalize the weight as

$$
w_{n}^{(j)}=\frac{w_{n}^{(j)}}{\sum_{j=1}^{M} \bar{w}_{n}^{(j)}}
$$

4. Estimate the mean and covariance by

$$
\begin{array}{r}
\mu_{n}=\sum_{j=1}^{M} w_{n}^{(j)} x_{n}^{(j)} \\
\Sigma_{n}=\sum_{j=1}^{M} w_{n}^{(j)}\left(\mu_{n}-x_{n}^{(j)}\right)\left(\mu_{n}-x_{n}^{(j)}\right)^{T}
\end{array}
$$

Using this method, we can predict where the group of people will be at certain times in the future. Moreover, once the prediction of people's motion behavior is computed, the group of robots can enable us to determine the locations on the path where people are most likely to stray.

\subsection{Anticipation}

Once the model has predicted the people's position, it must identify the potential conflict areas, determine where the robots should move in order to prevent straying and assign the robots positions in order to be able to guide the group of people.

Thus, the model is able to compute the optimal number of robots needed and structure of their formation so as to minimize the work they must perform. The presented model is applicable to any urban area. To study potential conflict areas, we have taken into account five different kinds of settings: open areas, entering a hallway, leaving a hallway, crossing intersections with three streets 
and crossing intersections with four streets, see Fig. 1. Other possibilities which may be considered are merely combinations of the above mentioned conditions.

In this paper, it is assumed the map on which robots perform their task it is already known as well as the path the group should follow. Therefore, the first step of the method consists of being aware of the open spaces (the areas in the map where there are no obstacles or their density is very low) along the planned path, as well as, hallways, open areas or intersections. After that, the obstacles are modeled as Gaussian functions using eq. 10 and, then, the probability map where the obstacles are the Gaussian functions and the rest of the map will be represented as a zero-function and can be computed. The function $H($ map $)$ is the occupied space of the obstacles and is calculated by adding all the Gaussian functions representing the obstacles:

$$
H(\operatorname{map})=\sum_{z \in \Omega} T_{z}\left(\mu_{z}, \Sigma_{z}\right)
$$

where, $\Omega$ is the set of obstacles in the environment, $z \in \Omega$, means that point $z$ is located inside an obstacle.

Moreover, we have also defined the robots as Gaussian functions, so, eq. 15 can be extended to include the robots as well:

$$
H_{\text {ext }}(\operatorname{map}, \text { conf })=\sum_{z \in \Omega} T_{z}\left(\mu_{z}, \Sigma_{z}\right)+\sum_{z \in\{\text { robots }\}} T_{z}\left(\mu_{z}, \Sigma_{z}\right)
$$

where conf is the set of robots' positions. In Fig. 4 there is an example of this representation. Therefore, robots can be seen as obstacles, since they can block the passage and act as a barrier.

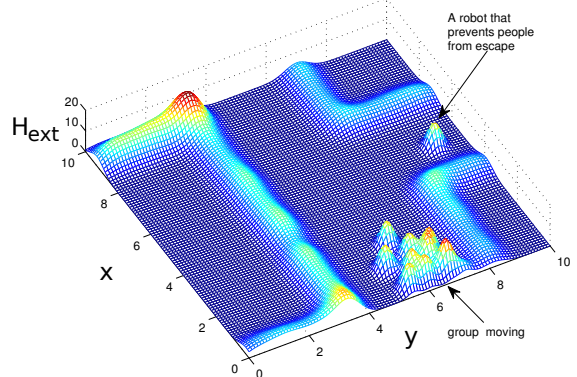

(a)

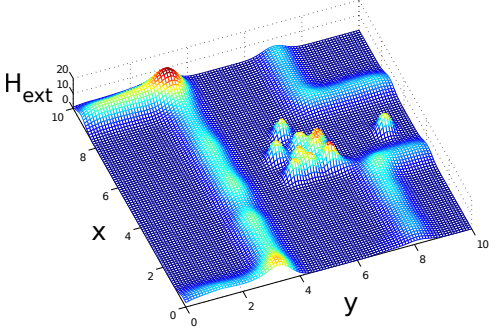

(b)

Figure 4: Graphical representation of eq. 16 using Gaussian functions of a group of people moving in an urban area. (a) The group starts moving, on the bottom of the y-axis, then the Prediction and Anticipation Model detects that one person can move away from the formation in the next corner, computes which robot can be sent and sends an auxiliary robot to that corner to prevent from people moving away. (b) The group passes along the crossing area but, as a robot is located in that area, people cannot move away. 
According to (Goldstone et al., 2006), when pedestrians move in groups, the people formation can be considered as rows of 2 to 4 people (the distances between them are shown in Table 1). The parameters of this table are: taking the values between brackets as the standard deviation error of the mean; $\alpha_{i j}$ is the angle between the walking direction of the person $p_{i}$ and the segment formed by person $p_{i}$ and person $p_{j}$; and $d_{i j}$ is the distance between $p_{i}$ and $p_{j}$.

Table 1: Average angle and distance values between group members for each group size (Goldstone et al., 2006)

\begin{tabular}{lccc}
\hline \hline & & $\alpha_{i j}(\mathrm{deg})$ & $d_{i j}(\mathrm{~m})$ \\
\hline size $=2$ & $p_{1} p_{2}$ & $98.8( \pm 1.12)$ & $0.78( \pm 0.02)$ \\
size $=3$ & $p_{1} p_{2}$ & $97.8( \pm 5.14)$ & $0.79( \pm 0.05)$ \\
& $p_{2} p_{3}$ & $87.1( \pm 4.46)$ & $0.81( \pm 0.10)$ \\
size $=4$ & $p_{1} p_{2}$ & $99.2( \pm 6.33)$ & $0.87( \pm 0.06)$ \\
& $p_{2} p_{3}$ & $87.7( \pm 6.54)$ & $0.93( \pm 0.09)$ \\
& $p_{3} p_{4}$ & $85.4( \pm 5.01)$ & $0.80( \pm 0.05)$ \\
\hline
\end{tabular}

Taking into account these measurements, if $N$ is the number of people who are being accompanied by robots, it can be assumed that group's width is $k *$ $d_{\text {side }}$, where $k$ is the number of people in a row, and $d_{\text {site }}$ is the distance between two people, see table 1 . Furthermore, the length of the group is $N / 3 * d_{\text {front }}$, where $d_{\text {front }}$ is the distance between one person and another who is in front of him/her. This measurement is defined by the personal space of a person described in section 4 .

Once the map of obstacles and the group size have been computed, the density of the obstacles along the path that the group will follow can be calculated. Since the group will move at a constant speed, we can compute at each instant $t$, the density of obstacles that a group of people will find along a specific path on the map. Thus, the function $F_{t}$ is computed as follows:

$$
F_{t}(\text { group_size, map })=\int_{x \in \Delta_{t}} H(x) d x
$$

where $\Delta_{t}$ is the space occupied by the intersection of the set of obstacles and group's position at time $t$. Therefore, $x \in \Delta_{t}$ means that the density of obstacles is computed in the area occupied by the group, see Fig. 5. Thus, for those areas where the density of obstacles is low, the probability that people will move away is high. Moreover, according to (Nourbakhsh et al., 2003), when people are being guided by a robot, for instance, in a museum, after 15 minutes, people's interest wanes, and about $26 \%$ of its members stray from the path. Therefore, in the present work, we consider that in free areas, up to $26 \%$ of the members can move away. If the value of $F_{t}$ is low in a specific area, it means there are many open zones and the robots must remain attentive to human reactions. Using this model, we can determine the optimal robots' motion behaviors in different environments for guiding purposes. 
Next, eq. 17 is extended to determine the configuration of the robots that best ensures that robots will cover the conflict areas -i.e., block the area in order to help people to remain in the group. We associate a scalar value with the group by using the function $F_{t_{\text {ext }}}($ conf, group), where conf is the set of positions of the robots and group is the position of the group of people being accompanied. This function indicates that the higher the value of $F_{t_{e x t}}$, the more control is needed. Thus, for the particular configuration of robots conf, the function $F_{t_{e x t}}$ will be:

$$
F_{t_{e x t}}(\operatorname{con} f, \text { map })=\int_{x \in \Delta_{t}} H_{e x t}(x) d x
$$

where $\Delta_{t}$ is the area occupied by the group of people and robots at time $t$. So, the configuration of robots that will be considered is the one that maximizes eq. 18:

$$
C=\operatorname{argmax}\left\{F_{t_{e x t}}(\operatorname{con} f, \operatorname{map})\right\}, \forall \text { configurations }
$$

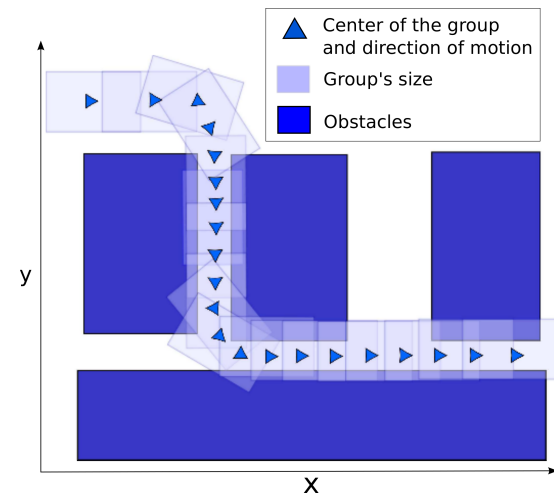

(a)

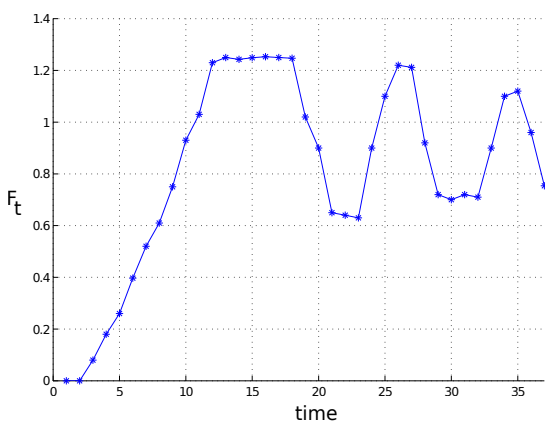

(b)

Figure 5: Graphical representation of a group of people moving through an area with obstacles and crossing areas. In (a) it is shown the path the group will follow, where the gray rectangles are the estimation of the group size, and the triangles are the mean position of the group and the direction of motion. In (b) the plot of $F_{t}$, which indicates the density of obstacles using eq. 17, is shown ; if the function's values increase, the density of obstacles in the area of the path is high, for example it can be seen that in corridors the value is high, however, in the crossing areas the value decreases as there are more free areas.

According to this strategy, the areas where people are likely to move away are covered. For groups of 6 or more people, the probability that more than one person will move away at the same place in different directions is high. For smaller groups, we only need to position a single robot in the open zones. 
That is, those zones which have a small value for the function $F_{t}$. However, for large groups, it is not enough to place just a single robot in such areas. As the robots know the map a priori, when they accompany large groups some might be positioned so as to form a barrier and prevent people from straying. Other strategies might be also used here, for example, a robot with an extensible arm or a rope might be employed.

Another issue to be determined is the best distribution of the robots to perform this task. In order to do that, we studied the work performed by the group. In the next section, we present a model that locally optimizes the work carried out by the robots in a way that achieved the minimum displacement of humans during a guiding mission.

\section{Optimal Robot Task Assignment for the Co- operative Mission}

In the previous section, we outlined how we determined the areas where people are more likely to move away depending on the density of obstacles along the path. The next step is to decide which robots should be sent to these areas depending on the group's motion and the position of the robots. In this section, we introduce a function based on the minimum robot energy (or work) criterion which determines the robots' optimal configuration (as it relates to the group of motion tasks assigned to each robot). We also tried to minimize the work performed by robots and achieve the minimum distances of displacement by the people. The following function can be used to solve two different tasks:

- Once the areas where people could stray have been identified, it must be determined which robots will be sent.

- If someone (a person or group of two people) leaves the group, it must be computed which robot should be sent to bring the person back to the formation.

In both cases, we identify the best strategy for the following situation: "Given a fixed number of robots (usually 2 or 3), assign robots' tasks which will minimize the work that will be required by them and, also, produce the minimum displacement problems for guiding people". Moreover, robots must be able to perform all their motion tasks while they navigate, avoid obstacles, and refrain from intruding on people's personal space.

In the case where we use two robots, one will be the leader and the second one will perform the task of regrouping and herding the people. If we use three robots, one will be the leader, and the other two will be used for regrouping or herding people. The leader of the robots is not predefined, in fact, the robots can change roles depending on the evaluation of the cost function. The robot tasks that have to be delegated in this stage are as follows: 
- Leader task: The leader robot computes a path planning and moves to the next point. We also assume that there exists a drag force which will attract people behind the robot. Here, the robot only has to move from the present position to the next one on along guiding path. If a robot that is not the leader assumes the leading role, this robot will have to move to the leader's present position before performing its task.

- Robot acting as a barrier task: As we explained in the previous section, it is more likely for people to become dispersed within certain areas on the map, therefore, in those areas where the density of obstacles is low, one or more robots should be sent to prevent people from straying. Here, it is assumed that the robots that perform this task well act as a repelling force pushing people to follow the group and preventing them from walking away from the formation.

- Looking for a person who goes away task: The robot moves to the estimated position of the person who has strayed from the formation. In this case, the robot must compute all possible paths to reach the estimated position and then assume the one which minimizes the itinerary. In our simulations, we used a selection of points on the environment where people are more likely to move away.

- Pushing task: The robot pushes a person who has strayed so he/she returns to the crowd formation. Since "push" has several meanings, the more direct one denotes the act of physically push a person, a more gentler method is to give instructions and accompany the person back to the group. This task can be also applied when a robot pushes a person (or people) who is (or are) following the formation in order to regroup people when the formation has fallen apart. We assume a repelling force which pushes the person to follow the robot's direction.

- Crowd traversing task: The robot has to move through the formation to achieve the estimated position of the person who strays from the crowd formation. This task implies that the robot has to push people away from their path, which creates a set of repulsion forces between the robot and the people. This particular situation is not addressed in the present work, due to safety concerns.

The cost function, described below, is computed based on the work performed, and can be divided into two blocks: (i) Robot work motion, and (ii) Human work motion.

In order to compute the dragging, pushing and crowd traversing forces, we use the equations defined in previous investigations on human behavior with other individuals (Helbing and Molnar, 1995). People's movements are determined by their desired speed and the goal they wish to reach. In our case, the direction of the person movement $\overrightarrow{e_{i}}(t)$ is given by:

$$
\overrightarrow{e_{i}}(t)=\vec{e}_{\text {robot }}(t)+\vec{u}(t)
$$


where $\vec{u}$ is the noise. Usually, people do not have a concrete goal and should follow the leader robot, thus, the person's direction is determined by the robot's movement or the person who they have in front of them, if the robot is not in their visual field.

In the following sections, we describe the different forces behind the computation of the cost function.

\subsection{Robot Work Motion}

Working with autonomous mobile robots, the robot $i$ work motion is expressed as:

$$
\begin{array}{r}
f_{i}^{m o t}=m_{i} a_{i} \\
W_{i}^{m o t}=f_{i}^{m o t} \Delta x_{i}
\end{array}
$$

where $m_{i}$ is the mass of the i-th robot, $a_{i}$ its acceleration and $\Delta x_{i}$ the space traversed by the robot to achieve its goal.

\subsection{Human Work Motion}

In Human Robot Interaction, it is necessary to consider the dragging, pushing and crowd intrusion forces produced by a certain robot's motion which can affect people. This component is called Human Work Motion, and it is the cost of people's movements as a result of robot's motions. As described earlier in this paper, the group follows the robot guide/leader, and a set of robots help to achieve their goal. The effect of robots on people as forces is as follows:

- Leader robot: attractive (dragging) force, inversely proportional to distance.

- Shepherding robot: Repulsive (pushing, traversing) force, which has a repulsive effect on people's personal space.

\subsubsection{Dragging Work}

The dragging force is necessary when the leader robot guides the group of people from one place to another. It acts as an attractive force, hence the force applied by robot leader $i$ to each person $j$ is:

$$
\begin{aligned}
f_{i j}^{d r a g}(t) & =-C_{i j} \overrightarrow{n_{i j}}(t)=-C_{i j} \frac{x_{i}(t)-x_{j}(t)}{d_{i j}(t)} \\
d_{i j}(t) & =\left\|x_{i}(t)-x_{j}(t)\right\|
\end{aligned}
$$

where $d_{i j}(t)$ is the normalized vector pointing from person $j$ to robot $i$ at instant $t$. See (Helbing, 1991) for more information about the parameter $C_{i j}$, which reflects the attraction coefficient over the individual $j$, and depends on the distance between the robot leader and person $j$. 
Thus, the dragging work, which the leader robot exerts on each individual, is defined by:

$$
W_{\text {drag }}=\sum_{\forall \text { person } \mathrm{j}} f_{i j}^{d r a g} \Delta s_{j}
$$

Where $\Delta s_{j}$ is the distance traveled by the person $j$.

\subsubsection{Pushing Work}

The Pushing force is understood to be the repulsive effect yielded by the shepherding robot on the group of people in order to regroup a person or prevent people from moving away by using a robot as a barrier. This repulsive force is caused by the robots' movements into people's personal space which is five feet around the humans body. This territorial effect might be described as a repulsive social force:

$$
f_{i j}^{\text {push }}=A_{i} \exp ^{\left(r_{i j}-d_{i j}\right) / B_{i}} \overrightarrow{n_{i j}}\left(\lambda_{i}+\left(1+\lambda_{i}\right) \frac{1+\cos \left(\varphi_{i j}\right)}{2}\right)
$$

Where $A_{i}$ is the interaction strength, $r_{i j}=r_{i}+r_{j}$ the sum of the radii of robot $i$ and person $j$, usually people have a radius of one meter and robots 1.5 $\mathrm{m}, B_{i}$ is the parameter of repulsive interaction, $d_{i j}(t)=\left\|x_{i}(t)-x_{j}(t)\right\|$ is the distance from the mass center of robot $i$ and person $j$. Finally, by choising $\lambda<1$, the parameter reflects the situation in front of a pedestrian, it has a larger impact on their behavior than things happening behind them. The angle $\varphi_{i j}(t)$ denotes the angle between the direction $\vec{e}_{i}(t)$ of motion and the direction $-n_{i j}(t)$ of the object exerting the repulsive force, see (Helbing, 1991).

So we can write pushing work by:

$$
W_{\text {push }}=\sum_{\forall \text { person in } \Omega_{i}} f_{i j}^{\text {push }}(t) \Delta s_{j}
$$

Where $\Omega_{i}$ is the set of people in which one of the shepherd robots has invaded the person's personal space. If an individual is more than two meters away from the robot, it is considered that the robot has not intruded on his/her personal space.

\subsubsection{Traversing Work}

And last but not least, the Traversing force is determined by the forces applied by the robot when traversing the crowd. For safety reasons, in this research, it has been decided the value of this force would be infinity to ensure that a robot would not cross the crowd or cause any damage.

\subsection{Total Cost for One Robot}

The cost function for robot $i$, given a specific task, is the following:

$$
W_{i}=\delta_{\text {mot }} W_{i}^{\text {mot }}+\delta_{\text {drag }} W_{i}^{\text {drag }}+\delta_{\text {push }} W_{i}^{\text {push }}+\delta_{\text {trav }} W_{i}^{\text {trav }}
$$




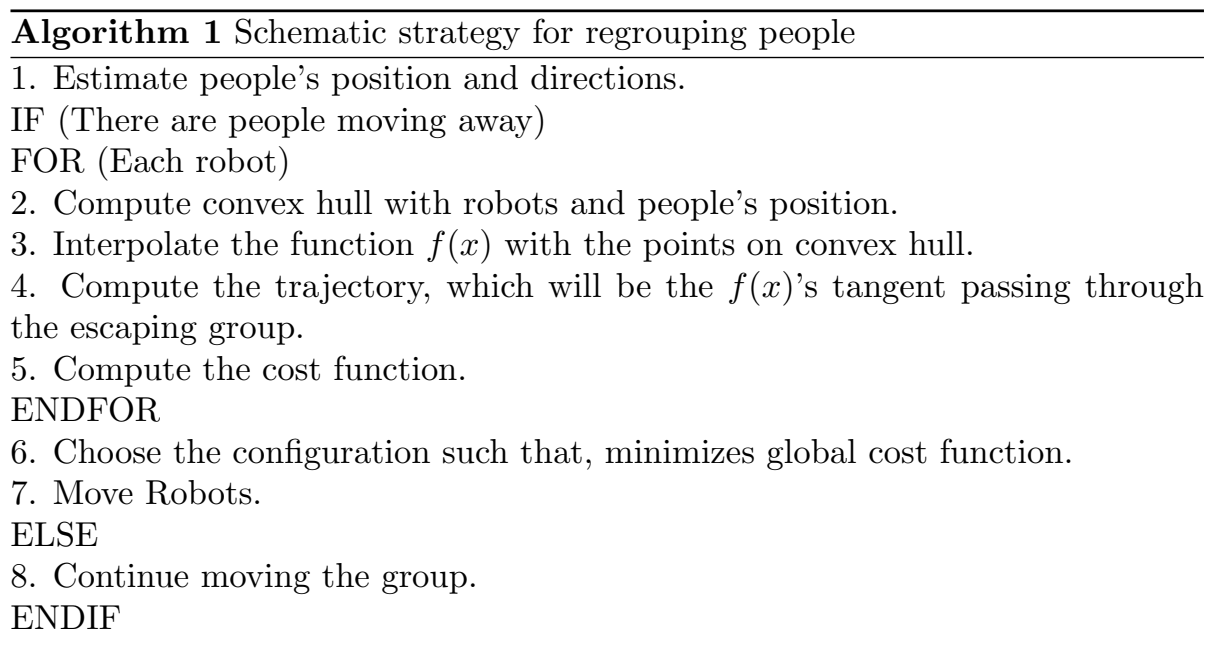

$$
\text { where } \delta_{k}=\left\{\begin{array}{l}
1 \text { if this task is assigned } \\
0 \text { if this task is not assigned }
\end{array}\right.
$$

Where $k$ could be pushing, dragging, traversing or motion. For each period of time, the leader and shepherd robots will be given a task in the guiding mission, which will require one or several robot work motions and human works motions.

\subsection{Local Optimal Robot Task Assignment}

At this point, we are able to compute a local optimal task assignment for the robots completing the guiding task. In this case, we mean "local" and not "global" optimal robot task assignment because we are only working with a short period of time ahead, when an event happens or can happen; for example, when a person strays from the group or where potential conflict areas in which people are more likely to stray are located. The local optimal robot task assignment will be one which minimizes the assigned work cost required to accomplish the local task, and it is computed as follows:

$$
C=\operatorname{argmin}\left\{W_{\text {total }}(c)\right\}, \forall \text { configuration } \mathrm{c}
$$

where the configuration indicates how the tasks are distributed among the robots, and $W_{\text {total }}(c)=\sum_{\forall c} W_{i}$ is the total cost for the $c$ robots that are working cooperatively. Eq. 29 computes the best configuration based on the minimum robot motion energy (or work) criterion.

Using $C$ we can identify the motion tasks for each robot and establish their trajectories in order to achieve their goals. A typical mission is to retrieve 
a person who has strayed from the group. In this case, the output of the configuration is that one robot has to reach the person who is moving away; once the robot has reached him/her it must apply a Pushing force to lead the person back to the group position. The scenario is adjusted in the case that several people move away in different directions at the same time. If there are enough robots, the model computes the robot's optimal configuration and assigns each one the goal of reaching the assigned person and leading him/her back to the group. If the number of people moving away in different directions is greater than the number of shepherd robots available, then, there is neither an optimal solution nor a good solution. This problem can only be resolved by the following means: when one shepherd robot is available, if the person is still in an area of the map where the robot can detect him/her, the best task assignment will be computed and robots will be set to achieve their goals. However, if this person cannot be detected, the problem has no possible solution.

Table 2 presents robots' tasks and behaviors, as well as, the forces that are applied in each case. The computation of the configurations and the procedure that should be followed by the robots is explained in the following section.

The various issues which emerge from the determination of this local optimal solution are as follows:

- The method employed has a time complexity of $N_{R}$ !, where $N_{R}$ is the number of robots used for guiding. Although we have not analyzed other faster methods (from the complexity point of view) in this work, there are various ways of reducing this time complexity using the environment or robot formation heuristics.

- The method finds a local optimal solution when the number of robots is greater than the number of persons who move away from the group. Otherwise, there is no local optimal solution.

- The method sequentially assigns the robots to follow the group of people who have strayed, or to prevent people from straying. If there are several configurations with the same cost function value, the system will automatically choose the first solution. In the case where there are two solutions where either the leader or a shepherding robot can be used to reach a person the method will select the latter.

- The scalability of the method depends on the number of people being guided and the number of robots present. If the number of robots and people are high, then due to the time complexity issue, the method might be too slow.

\section{Computation of the Robot Configurations for Group Reunification}

One of the most common problems which arises when robots guide a group of people occurs when one or more people move away from the group and need to 
Table 2: Tasks and Behaviors

\begin{tabular}{|c|c|c|c|}
\hline Task & Robot & Forces Applied & Behavior \\
\hline \multirow[t]{3}{*}{ Guide the group } & Leader & $f^{d r a g}$ & Act as a tour guide \\
\hline & Shepherd & $\begin{array}{l}f^{\text {drag }} \\
f^{\text {push }} \\
f^{\text {trav }}\end{array}$ & $\begin{array}{l}\text { Interchange } \\
\text { the role with } \\
\text { the robot leader }\end{array}$ \\
\hline & & $f^{d r a g}$ & Act as a tour guide. \\
\hline \multirow{2}{*}{ Join the group } & Leader & $f^{d r a g}$ & Reduce the velocity \\
\hline & Shepherd & $f^{p u s h}$ & Increase the velocity \\
\hline \multirow[t]{3}{*}{ Rescue people } & Leader & $f^{d r a g}$ & $\begin{array}{l}\text { All the group follows } \\
\text { the leader. }\end{array}$ \\
\hline & & $\begin{array}{l}f^{\text {drag }} \\
f^{\text {push }} \\
f^{\text {trav }}\end{array}$ & $\begin{array}{l}\text { Robot leader interchange } \\
\text { its role with shepherd } \\
\text { robots. }\end{array}$ \\
\hline & Shepherd & $\begin{array}{l}f^{\text {puch }} \\
f^{\text {drag }} \\
f^{\text {trav }}\end{array}$ & $\begin{array}{l}\text { Compute the } \\
\text { trajectory for } \\
\text { reconduct people. }\end{array}$ \\
\hline \multirow[t]{2}{*}{ Barrier in a cross } & Leader & $f^{\text {drag }}$ & $\begin{array}{l}\text { Follows the trajectory } \\
\text { till the goal }\end{array}$ \\
\hline & Shepherd & $f^{\text {push }}$ & $\begin{array}{l}\text { Robot moves toward } \\
\text { the corner and wait } \\
\text { for the group passes. }\end{array}$ \\
\hline \multirow[t]{2}{*}{ Narrow corridor } & Leader & $f^{d r a g}$ & $\begin{array}{l}\text { Follows the trajectory } \\
\text { till the goal. }\end{array}$ \\
\hline & Shepherd & $f^{\text {push }}$ & $\begin{array}{l}\text { Wait all the group } \\
\text { enters the narrow corridor }\end{array}$ \\
\hline
\end{tabular}

be retrieved, either because they are interested in an object lying outside the trajectory of the group, or because they become distracted. In this section we describe the method of reintegrating people who have strayed from the group using the cost function described above.

When this problem occurs, the robots must adjust their goals. For instance, one of the shepherd robots can change its direction, instead of following leader's trajectory, or the leader robot can become a shepherd robot. Therefore, it is necessary to evaluate the cost and the consequences of such changes in robot roles and trajectories. In Table 2 we describe the robots' forces and behavior in each guiding task.

In order to compute the best configuration $C$ which minimizes the total robot cost in a regrouping task, the system must predict, for each configuration of robots, people's positions and motion vectors (Arulampalam et al., 2002), as well as how much work each robot must do to perform its task. People's motion 


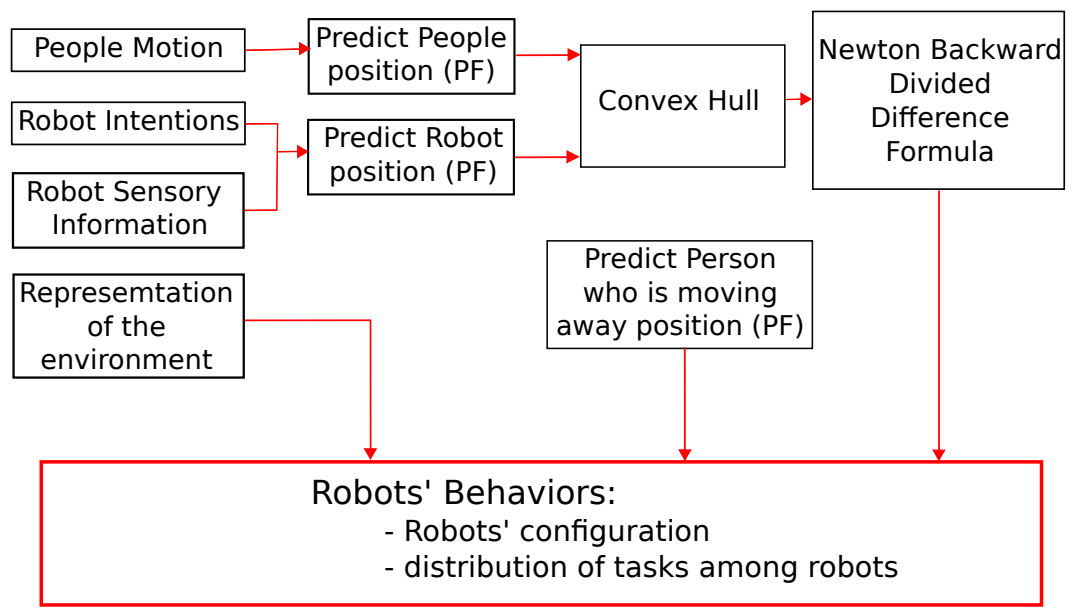

Figure 6: Procedure for calculating the path that a robot should follow to the position of the person who is moving away. Using the particle filter the positions of robots and people are computed. Considering the position of the group of people who are following the leader and the robots which are accompanying the group, the convex hull is computed (here, the person who is moving away is not considered, and the robot which will develop this task neither). Then, the function which interpolates the points in the convex hull is computed using Newton Backward Difference Formula. Finally, the trajectory the robot should follow is: firstly, the tangent function of the function $\mathrm{f}(\mathrm{x})$ and passing through this robot, and, secondly, the tangent function of the function $f(x)$ and the person who is moving away.

is predicted with a particle filter which considers all the positions of people and robots as well as the obstacles in the environment. To compute the work that each robot must carry out, eq. 28 , the system has to compute the robot work motion, and the robot work required by Helbings' forces. The robot work motion must take into account the trajectory the robot has to take in order to reach the person who is moving away. There are various trajectories the robot has to perform: $(i)$ if the person who is moving away is within the robot's field of vision, and there are no obstacles, then the trajectory is a straight line; $(\mathrm{ii})$ if the person who moves away is on the same side of the robot, but there are obstacles, then the trajectory must account for these obstacles, see (Garrell and Sanfeliu, 2010a); ( iii) if the person who strays is on the opposite side of the group of people being guided, in relationship to the robot, then the robot has to first to determine the proper trajectory to go around the group of people and then compute the trajectory depending on whether it is more similar to case $(i)$ or case $(i i)$.

A discussion of the second part of case (iii) can be found in the article (Garrell and Sanfeliu, 2010a), so here we shall only explain the first part of case ( $\mathrm{iii}$ ) here. In order to compute the trajectory in order to go around the group of people, we must calculate the convex hull of the people's and robots' positions. 
If we treat the group of people moving away in the same direction as a single element, then we might use its arithmetic center as the position of the group. Next, the function that interpolates the points in the convex hull is computed for each robot using the Newton Backward Divided Difference Formula, which consider only those people in the area between the robot, the convex hull and the group that is moving away, such that we arrive at the function $f(x)$, see Fig. 6. Then we compute the trajectory of the robot, denoted as the tangent of $f(x)$, that passes through the center position of the moving group. This procedure is computed each interval of time $k$ until the robot arrives at the moving group and the group is redirected toward the formation to be followed, see Fig. 6. To compute the total amount of required work we compared different trajectories and selected the one that yields a lower cost function.

In the simulation section of this paper, we present the results of these cost function calculations, and describe a comparative study of different trajectories. In algorithm 1 we introduce a schematic procedure to be followed by the group of robots.

\section{Real-life Experiments to Verify Human Reac- tive Behavior}

The aim of this study is to describe how a group of robots working cooperatively can effectively accompany a group of people. We focus on two key issues: preventing people from straying from the formation and studying the behavior of the team of robots when one or more people of the group move away.

In this section, we demonstrate that the Helbing et al. model of forces is applicable to our study. To verify these forces, we have conducted a series of real-life experiments using our own robot, called Tibi, see Fig. 7, and a group of volunteers who had not worked with service robots previously. We selected this group of volunteers so that they had no prior contact with robots and had not been conditioned by them. We conducted a set of experiments in which the robot approaches a person from different angles and at different speeds. The robot was controlled by teleoperation, but the volunteers were not aware of this fact. The results of these experiments enabled us to determine the personal space a person requires when he/she interacts with a robot, and allowed us to assess the effects of pushing and dragging forces on human individuals.

\subsection{Tibi Robot}

Two mobile service robots, Tibi and Dabo, designed to operate in urban pedestrian areas, were developed in the European URUS (Ubiquitous Networking Robotics for Urban Sites) project (Sanfeliu and Andrade-Cetto, 2006). Tibi, pictured in Fig. 7, is based on the two-wheeled, self-balancing Segway RMP200 platforms. The Segway RMP200 is, in many ways, an ideal platform to run in urban areas. Humanoid robots are not yet equipped to operate in outdoor environments. 

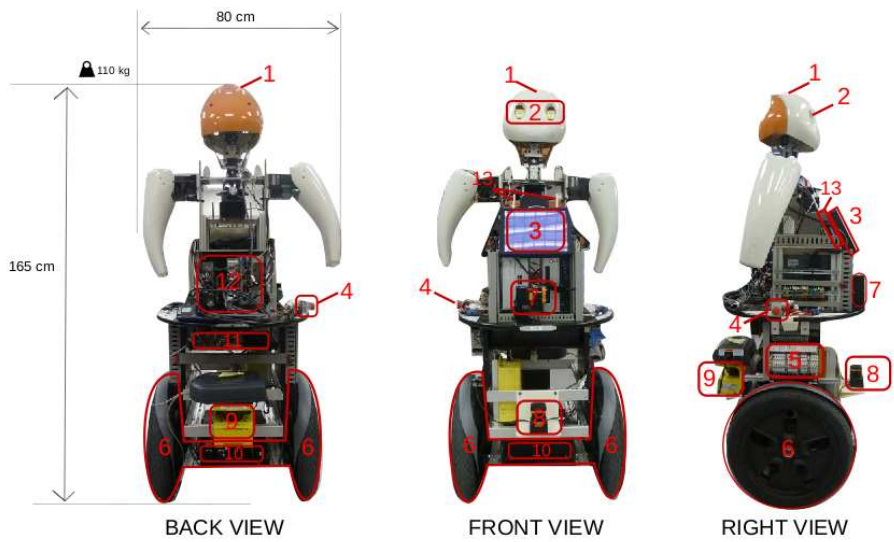

Figure 7: Components of the Tibi Robot: (1) the head; (2) Bumblebee stereo cameras; (3) monitor; (4) emergency stop button; (5) maneuvering buttons; (6) Segway wheels; (7) vertical front laser for navigation; (8) horizontal front laser for navigation and localization; (9) horizontal back laser for navigation and localization; (10) Segway batteries and controller; (11) additional batteries; (12) on board computers; (13) wireless antenna.

The Tibi robot has a height of $165 \mathrm{~cm}$, occupies a clearance space of $80 \mathrm{~cm}$, and weights $110 \mathrm{~kg}$. It is equipped with multiple sensors, as well as, a Bumblebee stereo camera and three lasers. To detect people in these experiments we used the front and back lasers located $40 \mathrm{~cm}$ from the ground.

The Tibi robot was designed to interact with different people in open spaces. The robot is socially accepted, and humans take an interest in interacting with it, robot's design must be well-rendered, and, its movements should be smooth. Moreover, it should know the personal space required by humans, so as to avoid invading on it and causing a negative reaction in people with whom it interacts.

Below, we present the methods and results of a series of experiments conducted with the robot Tibi. These experiments have been designed to analyze people's reactions when Tibi approached them (push force), when they walked together (push force) or when a person followed a robot (dragging force).

\subsection{Experiment Structure and Design}

To perform the experiments, we had the participation of fifteen volunteers aged from 20 to 40 years old. We performed two different sets of real-life experiments: the first set aimed to obtain the persons' personal space preferences when the individual stood at a certain point and was approached by the robot; the second set was designed to determine the distances between the robot and the person for the dragging and pushing forces. In the first set, the person stood at a certain point and the robot approached him/her at different speeds and angles, when the person felt the robot too close, he/she could start walking away. This reaction manifests the parameters of the person's preferences for personal space 
when he/she interacts with the Tibi robot.
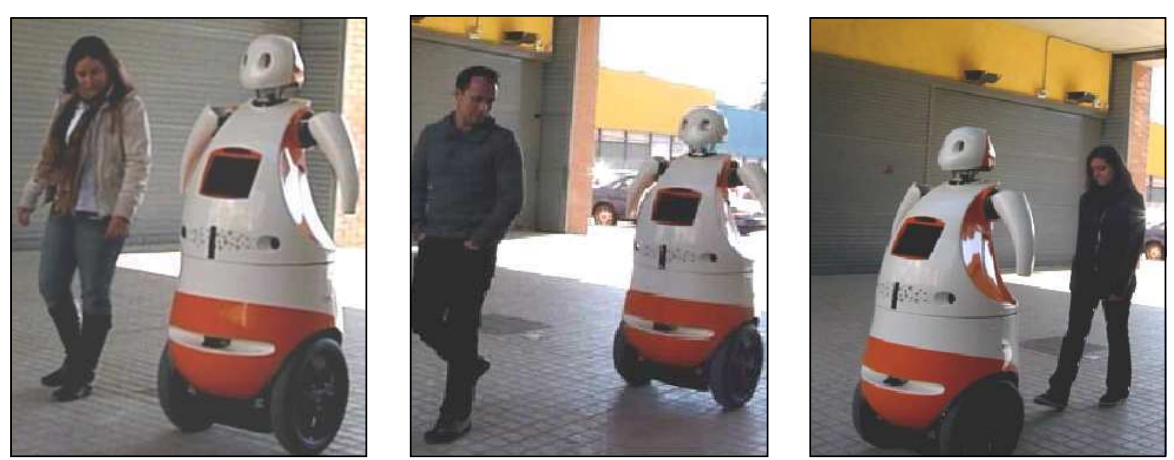

Figure 8: Three different people walking with Tibi Robot. Left: Tibi walks side by side with the volunteer. Center: Robot walks behind the person pushing him/her (the Helbings' pushing force is applied). Right: Tibi walks in front the person and, here, the dragging force is applied.

In the second set of experiments, we analyzed the behavior of people when accompanied by Tibi. In this set of experiments we measured the distances between the robot and the person when the pushing forces or the dragging forces were applied in three distinct situations. In the first scenario the robot was the leader and the person followed the robot propelled by the dragging force; in the second, the robot was located behind the person and forced the person to move via the pushing force; and in the third, the robot was located at the person's side and forced him/her to move via the pushing force. Here, though the robot and the human have followed the same path, Tibi moved at different speeds, and it performed the role of the leader or shepherd, see Fig. 8.

The results and conclusions of the set of experiments are described in the following subsection.

\subsection{Human Behavioral Responses to Robot Motions}

As mentioned above, the first part of the experiments performed aimed to determine the personal space desired by people when they interact with the robot. To that end, we conducted a series of tests in which the robot moved towards a person at different speeds $(0.3 \mathrm{~m} / \mathrm{s}, 0.6 \mathrm{~m} / \mathrm{s}$ and $0.9 \mathrm{~m} / \mathrm{s})$ and at different angles $\left(0^{\circ}, 45^{\circ}, 90^{\circ}, 135^{\circ}\right.$ and $\left.180^{\circ}\right)$.

Fig. 9 shows the distances between the robot and a person, where different speeds and angles were considered. Note that as speed increases the distances become greater, and the size of the personal space increases proportionally. The distances also vary, depending on the angle of approach. The results are summarized in Fig. 10, which shows the size of personal space desired, depending on the robot's velocity.

In the second part of the experiments, we evaluated the effect of the leader robot and the shepherd robot when they accompanied people. We also studied 

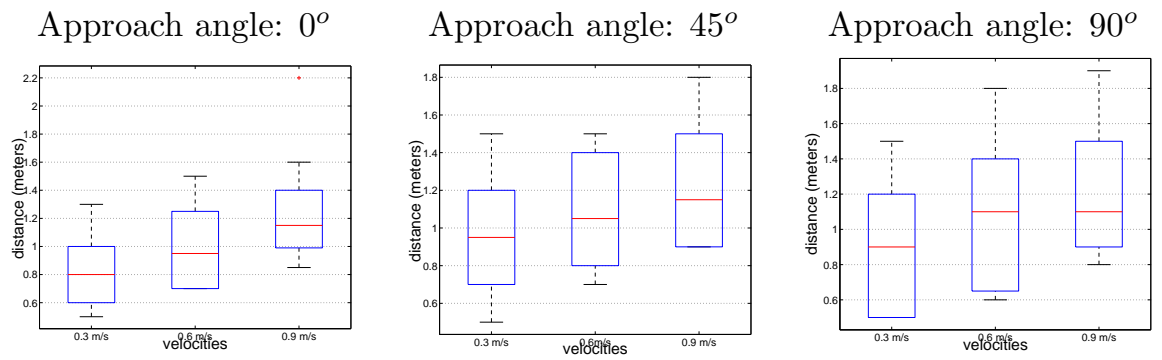

Approach angle: $135^{\circ}$

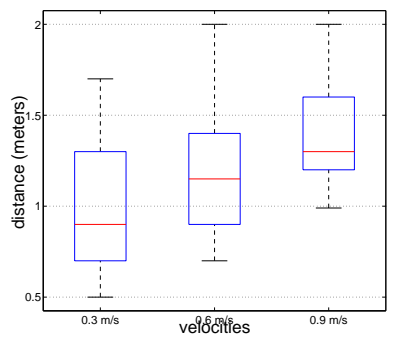

Approach angle: $180^{\circ}$

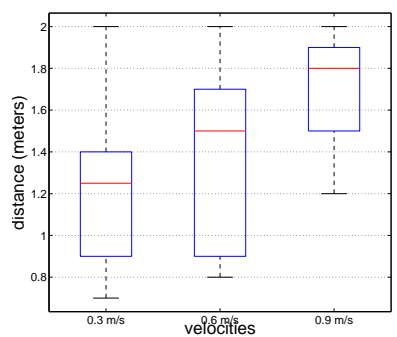

Figure 9: First set of experiments: the person stands at a certain fixed point and does not move up until the robot is too close. The graphs show the distances between the robot and the person (y-axis) at different speeds ( $\mathrm{x}$-axis) before he/she moved due to the robot is too close. The graphs show the mean, variance and quartiles of the data obtained in the experiments at different approach angles.

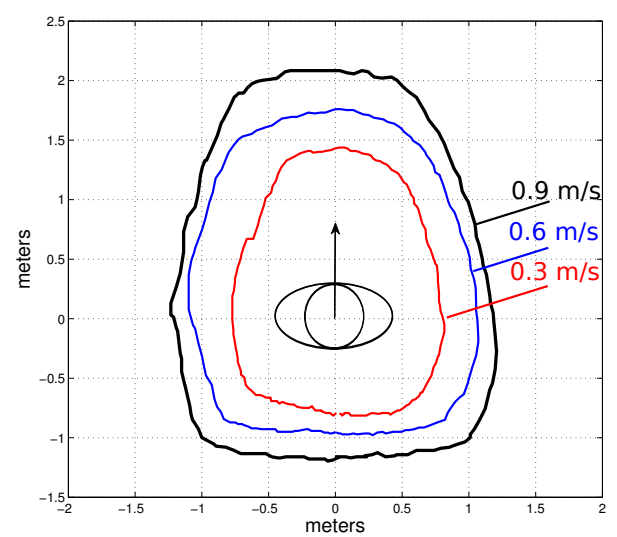

Figure 10: Summary of the personal area of a person provoked by the approach of the Tibi robot at different speeds of robot motion.

the participants' behavior when they were accompanied by Tibi. In the first situation, the robot assumed the role of the leader, and the participants had 
to follow him. Fig. 11 shows the distances between the person and the robot along the way. The robot accelerated and decelerated, the remaining distance is constant, within certain parameters. This shows the force of attraction of the leader and suggests that people follow the path of the leader.

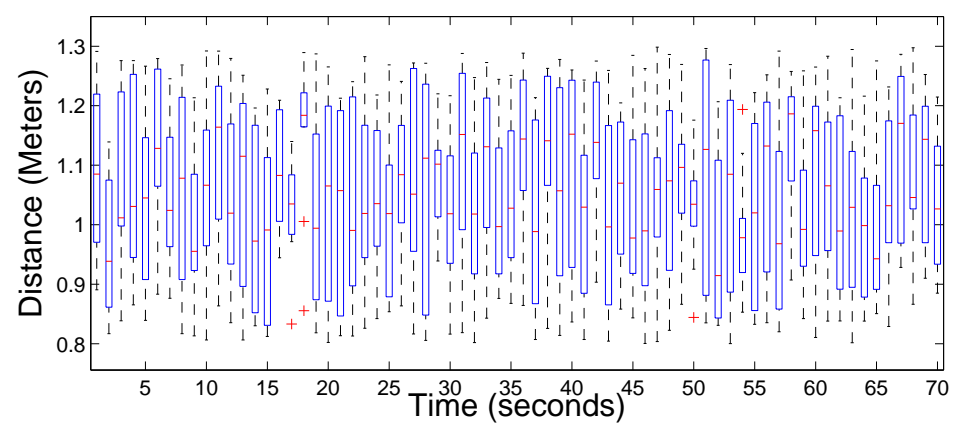

Figure 11: Second set of experiments: the robot is the leader and the person follows the robot provoked by the dragging force. The graph shows the distance between the leader robot and people (y-axis) during a period of time (x-axis) where the robot accelerated and decelerated. It can be observed that the distances varied between $0.85 \mathrm{~m}$ to $1.27 \mathrm{~m}$.

In Fig. 12 the different results that were obtained are presented: the distance between a volunteer and the robot, and the robot and a volunteer's speed and acceleration while Tibi performed the leader role. It is shown that during the process of guiding the distances were maintained and the human attempts to imitate the velocity of the robot, and therefore, if the robot accelerates the person increases its speed, and analogously if the robot reduces its velocity.

Lastly, we studied people's behavior when the robots played the role of shepherd. In this scenario, Tibi shifted to people's sides or positioned itself behind them while moving at different speeds. In Fig 14, the robot was positioned behind people. When the robot's speed was too slow, $0.4 \mathrm{~m} / \mathrm{s}$, the human's
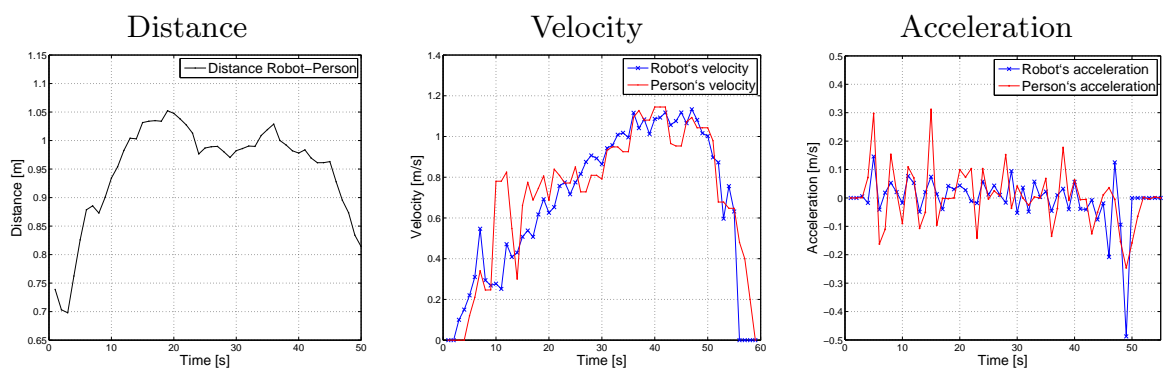

Figure 12: Robot acts as a leader while a person follows it. Distance, velocity and acceleration of the robot and the person during the guiding process. 

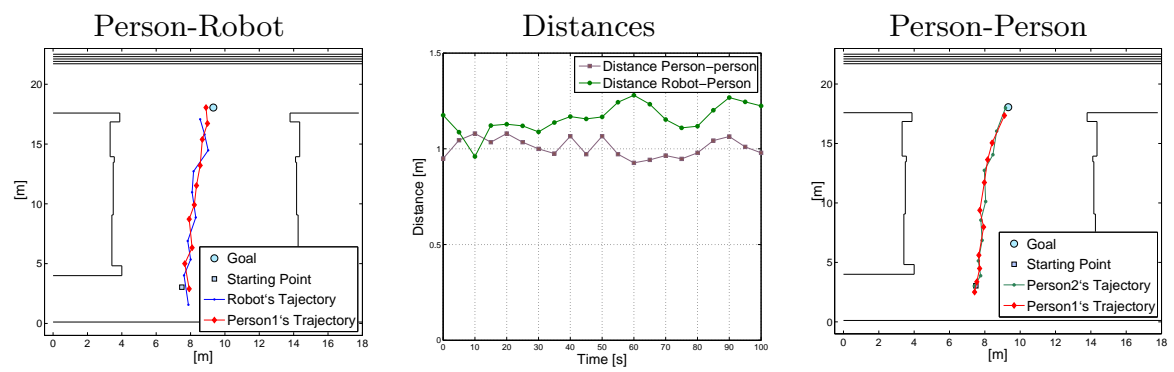

Figure 13: Comparison of the trajectories: Left: Robot navigates behind a volunteer. Center: Distances between the volunteer and the robot (or another person). Right: A person walks behind the volunteer.

interest decreased and the person strayed from the robot. However, if the robot moved faster, at $0.8 \mathrm{~m} / \mathrm{s}$, the person felt that the robot was pushing him/her and he/she tried to keep the distance constant.

According to (Bohannon, 1997), the mean comfortable speed ranges from $1.272 \mathrm{~m} / \mathrm{s}$ to $1.462 \mathrm{~m} / \mathrm{s}$, for this reason, we do not allow the robot to reach speeds greater than $1 \mathrm{~m} / \mathrm{s}$. When volunteers were asked if they felt comfortable with a robot walking behind them at speeds close to $0.8 \mathrm{~m} / \mathrm{s}$, they said they felt safe and walked until the robot stopped moving. In addition, we conducted a study comparing the behavior of the volunteers when they have a robot behind them or another person following them. Fig. 13 shows the trajectories performed in both cases and the distances measured during the path. It was found that if the speeds are between $0.7 \mathrm{~m} / \mathrm{s}$ and $1 \mathrm{~m} / \mathrm{s}$ behaviors are very similar.

Finally, Fig. 15 shows that when the robot walks side by side with the person, the distances are smaller, probably because people have less safety concerns in the company of the robots. Also, in this situation, the robot is accompanying the person instead of pushing him/her.

In conclusion, we computed the personal space desired within the company of our robot Tibi, and we analyzed the effects of the dragging and pushing forces, depending on robot's movements and depending on robot's role, as a leader or shepherd. These observations were then incorporated into our simulations to verify the functionality of our model. The following sections present the simulation results in light of the values obtained in these experiments.

\section{Simulations in the Barcelona Robot Lab}

In this section, we explain two series of three simulations conducted using the PAM model. The first set of simulations was conducted using the map of the Barcelona Robot Lab. The second set focused only on analyzing the regrouping task. The simulations used the map of the Barcelona Robot Lab which measures about $10000 \mathrm{~m}^{2}$. This urban area is located in the North Campus of the Universitat Politècnica de Catalunya (UPC). The urban area includes corridors, 

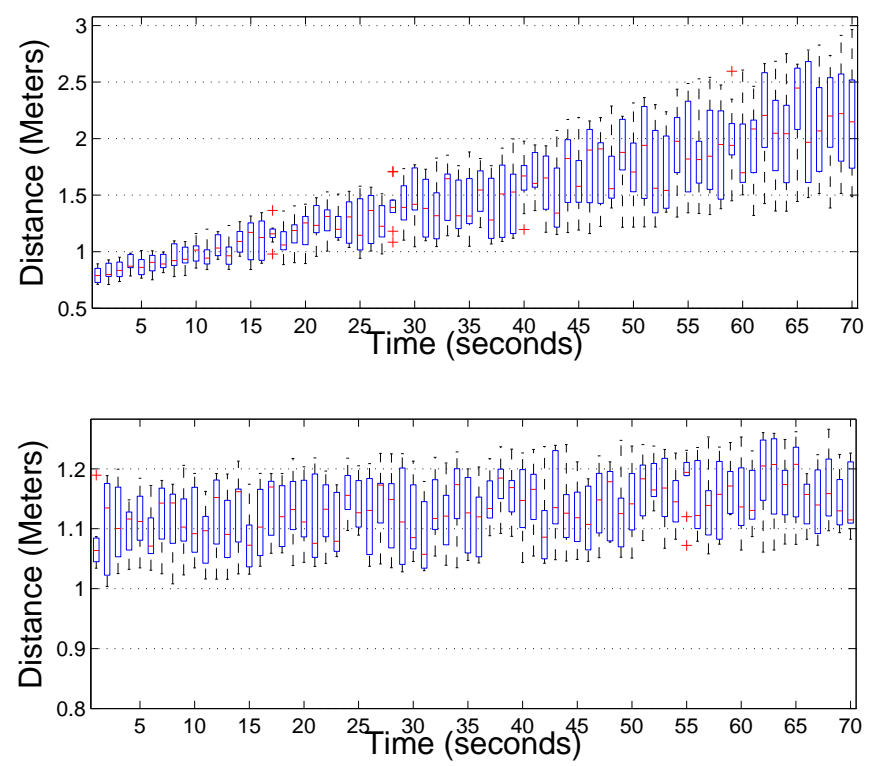

Figure 14: Second set of experiments: the robot is located behind the person and forces the person to start moving due to the pushing force. The graphs show the distance between the shepherd robot and people (y-axis) during a period of time (x-axis). It can be observed in the top graph that, if the robot speed is too slow $(0.4 \mathrm{~m} / \mathrm{s})$, persons' interest decreases and the person does not feel the robot is pushing and then he/she starts moving independently of robot speed - the distance between the robot and the person increases during the walking period. If the robot speed is higher $(0.8 \mathrm{~m} / \mathrm{s}$ ) (see bottom graph), then the person feels that the robot is pushing him/her and the person tries to maintain a constant distance between them.

open areas and intersection areas, as well as, static obstacles (buildings, benches or potted plants), and dynamic obstacles (mobile robots and people in motion). We then compared the results from the simulations conducted with and without the PAM model.

The simulations dealt with groups of people who followed the models described by Helbing et al. (Helbing and Molnar, 1995); additionally, we assumed the participation of a group of two or three robots which move according to the Discrete Time Motion Model (DTM) (Garrell et al., 2009), and behave according to the computation of the configurations explained in Section 7. 


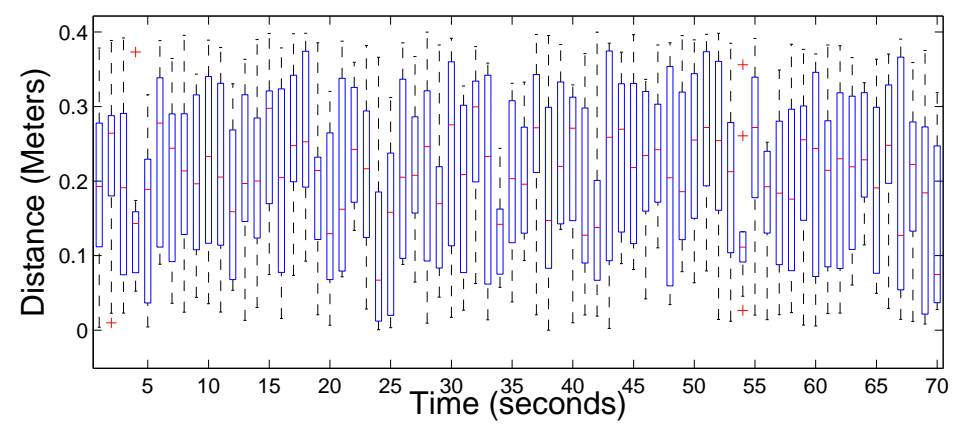

Figure 15: Second set of experiments: the robot and the person are side by side, and the person walks due to the pushing force. The graph shows the distance between the shepherd robot and people (y-axis) during a period of time (x-axis). Notice that distances between the robot and the person are between $0.2 \mathrm{~m}$ and $0.37 \mathrm{~m}$.

\subsection{Simulations using the PAM model}

We performed several simulations in the map of the Barcelona Robot Lab in which two or three robots guided a group of people. The robots were supposed to reach a specific target position in the map and react to unexpected situations. For each simulation, we compared the results with and without the PAM model described above. With the PAM model the simulated robots were able to anticipate the problem of people moving away and avoid losing a member of the group. If the robots do not use this model, then, the time of regrouping was much higher because they had to look for the people who had moved away, instead of preventing them to leave the group.

In the first simulation, two robots guided a group of five people along a path where there were two areas where people could move away, see Fig 16. We compared whether the robots behave in accordance with the Prediction and Anticipation Model or not, where the behavior of people who do not move away remains constant. Fig 16-Top shows that when the PAM model is used the group follows the path and nobody leaves the formation, but where the PAM model is not applied there are two areas where people move away and robots must look for them and bring them back to the formation. In Fig 16-Center, the covariance of the size of the group is plotted. Note that, if the robots are able to anticipate human motion behavior, they can prevent them from straying and, thus, maintaining the covariance of the group. However, if the robots have to wait for someone to stray before taking action, the size of the covariance grows.

Anticipating or expecting someone to stray, can be seen on the graphs that show the work required by the robots (and people) to maintain the group of people on the desired path. In other words, the work required by the robots to look for one or more people who stray from the group, is much lower if robots can anticipate and help people to follow the path by avoiding dispersions, see 
Fig 16-Bottom. Also note that in this visual aid the two peaks of the work performed, correspond to the two instants of time in which a person leaves the group and must be accompanied back.

In the second simulation, three robots guide a group of seven people along a path within an area where people can move away in different directions, see Fig 17. Again, we used this scenario to compare trends in the robots' behavior with and without the application of the Prediction and Anticipation Model. In both cases, the behavior of people who did not move away remained constant. In Fig 17-Top it is demonstrated that when the PAM model is used, the group follows the path and nobody leaves the formation. However, where the model is not used, there is an area in which two people move away in different directions and the robots must look for them and return them to the group. In Fig 17Center, the covariance of the group is plotted, and it can be seen that the covariance is larger when the PAM model is not employed.

Fig 17-Bottom represents the work performed by the group, where it can be observed that the work is lower when robots can anticipate stray movements and help people to follow the path by preventing dispersions. Note that the peak of the function corresponds to the moment in time in which two people leave the group and they must be accompanied back through the deployment of two shepherd robots.

In the third simulation, three robots guide a group of seven people along a path within an area where people can move away in different directions, see Fig 18. Again, a comparison is made between the robots' behavior with and without the employment of the Prediction and Anticipation Model. Fig 18-Top demonstrates that when the PAM model is used, the group follows the path and nobody leaves the formation; however, where the model is not used, there is an area in which two people move away in different directions and the two shepherd robots must look for them and return them to the group. Fig 18-Center plots the covariance of the group.

In Fig 18-Bottom, the work performed by the group is presented. It shows that the work is lower when robots are able to help people follow the path and preventing dispersions. It must be mentioned, although there are no shepherd robots in the back of the formation, people are still attracted by the humans who they have in front, and, thus, they follow the group and do not disperse.

In summary, we may conclude that, through the application of the prediction and anticipation model, robots are able to help people to follow the path, managing to prevent people from straying while carrying out their work at a lower cost.

(videos available in http://www.iri.upc.edu/people/agarrell/ijrr12.html)

\subsection{Simulations of Regrouping People}

In this subsection, we analyze the performance of the regrouping task, through three simulations conducted in open areas and at intersection.

In the first simulation, two robots guide a group of five people in an open area without obstacles, see Fig. 19. The positions of the two simulated robots are 

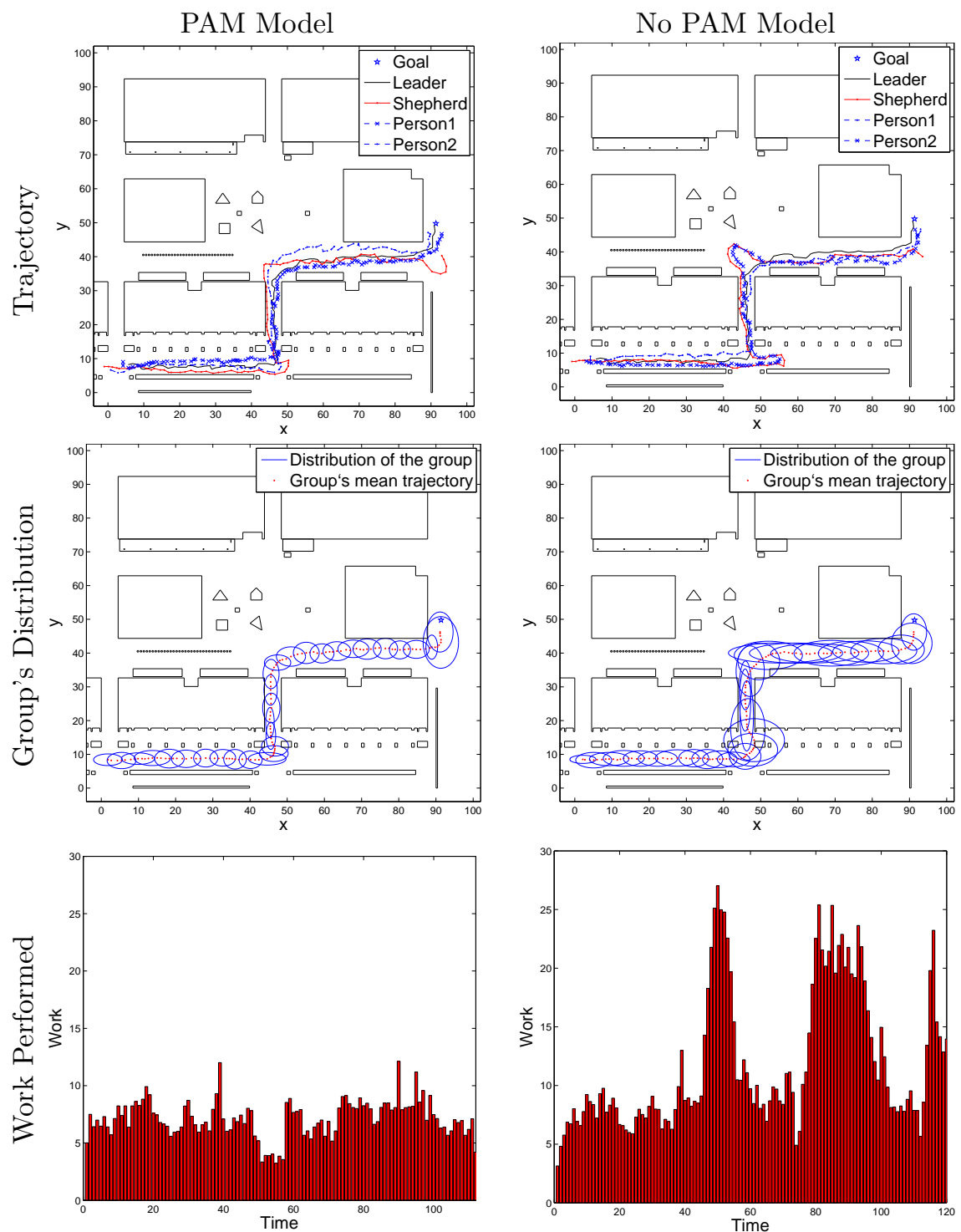

Figure 16: Simulation \#1: Graphic summary of people and robots' path for the first simulation, using the Prediction and Anticipation model (left), and without considering it (right). Top: Final trajectories of the simulated people and robots. Center: Trajectory of the whole group, shown as mean path and covariance. The increase of the covariance size is produced when a person leaves the group. Bottom: Graph of the Work performed by the group. 

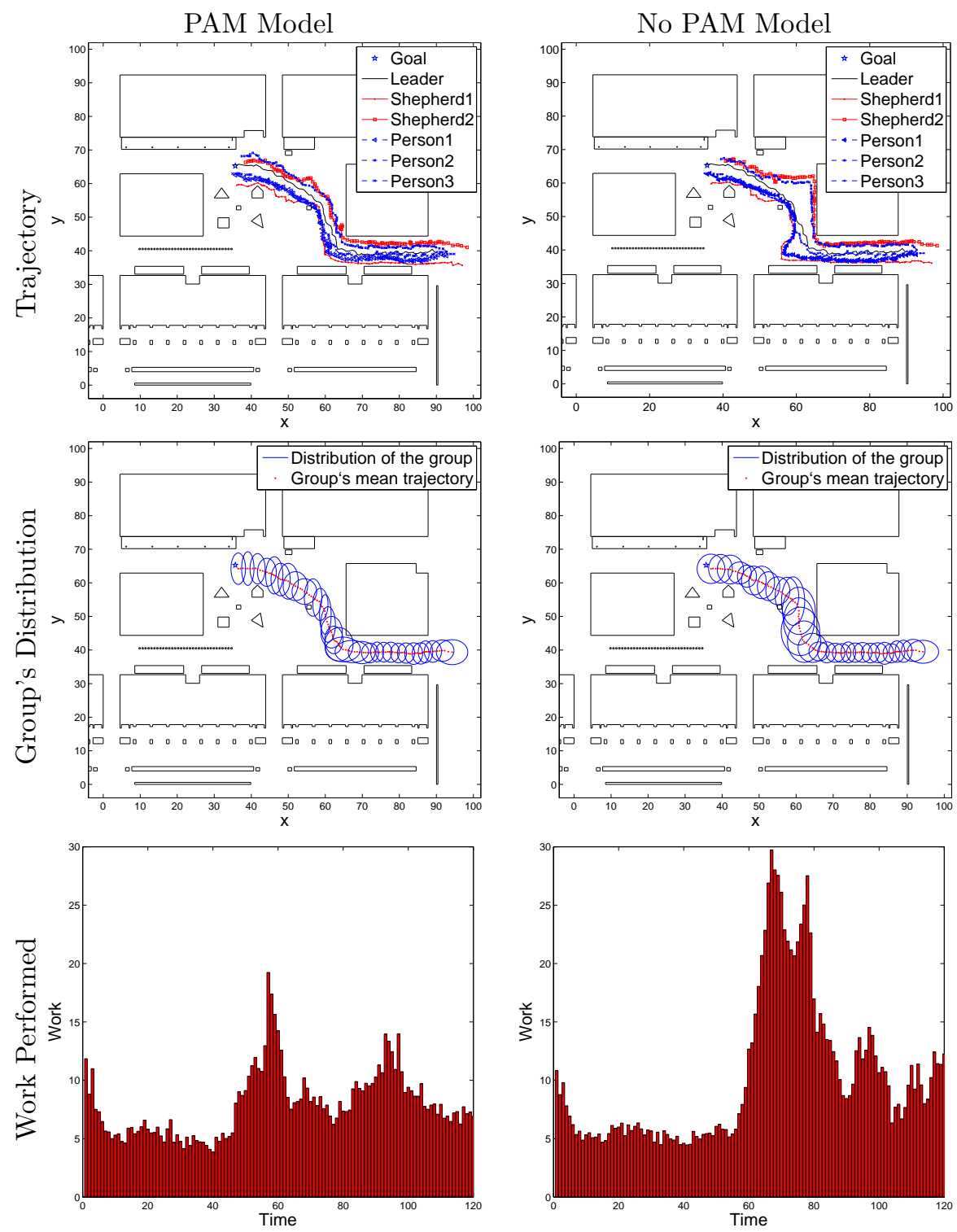

Figure 17: Simulation \#2: Summary of people and robots' path for the second simulation, using the Prediction and Anticipation model (left), and without considering it (right).

plotted with circles, the placement of the five people is indicated by asterisks. As mentioned above, when the robots detect that people are moving away, or an open area exists that may lead to dispersion, they must determine the 

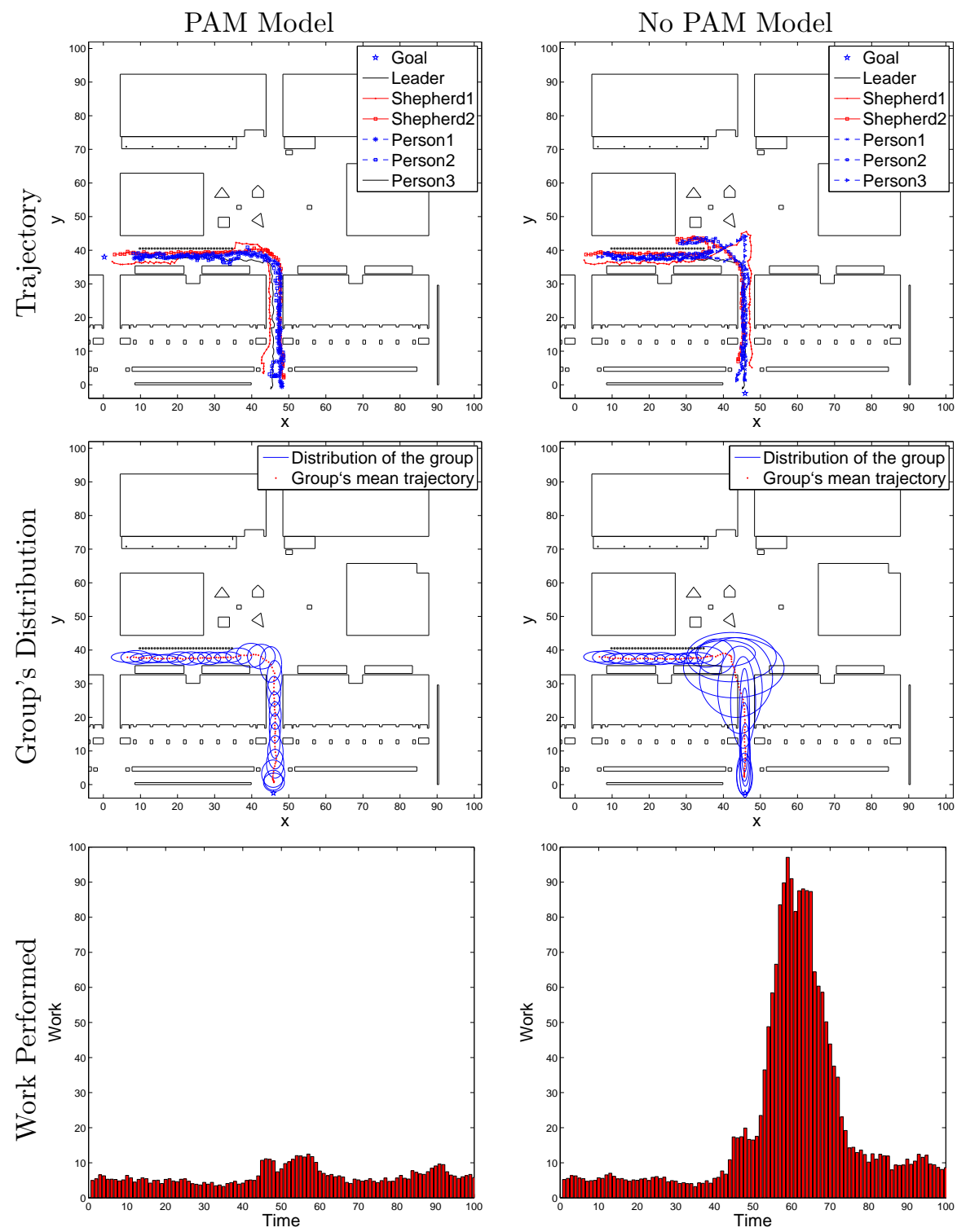

Figure 18: Simulation \#3: Summary of people and robots' path for the third simulation, using the Prediction and Anticipation model (left), and without considering it (right).

optimal configuration of robots to perform the task of returning the people to the group and/or preventing them from straying. The following are the possible configurations for regrouping people with two robots, one leader and 

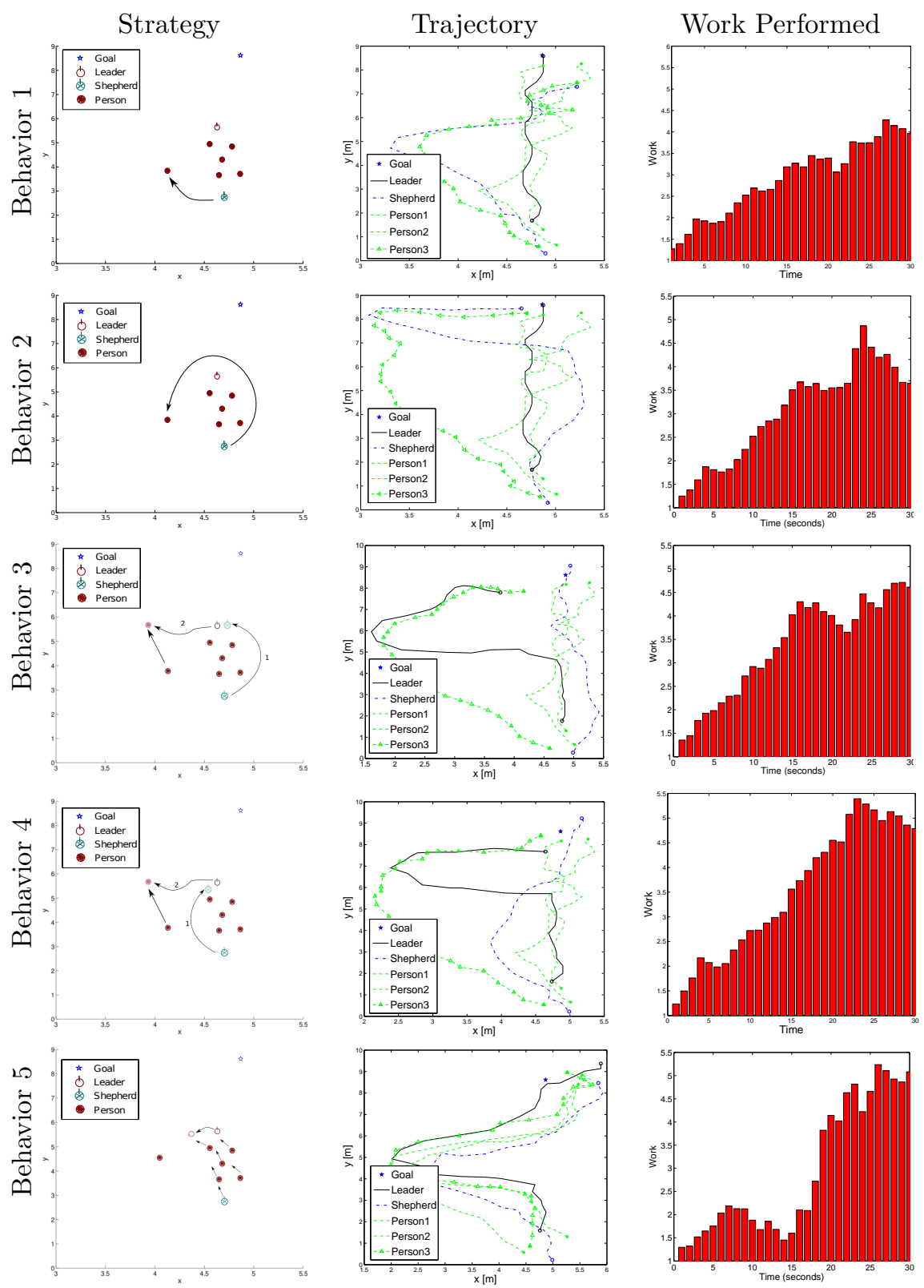

Figure 19: Simulation of the Regrouping \#1: Left: Different strategies that robots can follow. Center: Trajectories the group follows. Right: Work performed by the group. 
one shepherd: (i) The shepherd robot takes care of regrouping people who have moved away following the left path Fig. 19-1st. (ii) Shepherd robot takes care of regrouping people who have moved away following the right path Fig. 192nd. (iii) and (iv) the shepherd robot takes the role of leader, left and right trajectory respectively, and the leader robot moves toward the person who has strayed. In all four cases, we assume that the shepherd robot is able to explain to the group that it has assumed the role of the leader Fig. 19-3rd and 4th. (v) The leader robot regroups the formation, and thus the entire group moves toward the person who has strayed Fig. 19-5th. For all five possible configurations the system computes the work cost, Fig. 19-right. Note that the first configuration is the one which requires the lowest work cost.

In the second simulation, three robots guide a group of eight people in a street crossing area, see Fig. 20. In this case the simulated robots must regroup two people who stray towards different directions at the same time. The following are the possible configurations for regrouping two different people with two shepherd robots and one leader: (i) the shepherd1 robot takes care of regrouping person1 and the shepherd 2 robot takes care of regrouping person2, Fig. 20-1st. (ii) The shepherd2 robot takes care of regrouping person 1 and the shepherd1 robot takes care of regrouping person2, Fig. 20-2nd. (iii) The shepherd1 robot assumes the role of leader, while the leader robot moves toward person1, and the shepherd2 robot regroups person2. (iv) The shepherd1 robot assumes the role of leader, the leader robot moves toward the person2, and the shepherd2 robot regroups person1. (v) The shepherd2 robot assumes the role of leader, the leader robot moves toward the person2, and the shepherd1 robot regroups person1. (vi) The shepherd2 robot assumes the role of leader, the leader robot moves toward the person1, and the shepherd1 robot regroups person2. (vii) The leader robot regroups the formation and the entire group moves toward the people who have strayed. (viii) The leader robot regroups the formation and the entire group moves toward people who have strayed. It should be mentioned that in this specific example, situations (iii)-(viii) cannot be considered, because if the leader robot moves toward the people who are moving away, it implies that it would have to cross through the group, but we have already established that the transverse force has not been considered in these experiments.

Finally, there are strategies by which we may compute the cost of looking for a single person. When the person has rejoined the group, the work cost is recalculated to accommodate the regrouping of the second person. This strategy implies that the two shepherd robots are not going to look simultaneously for all the people moving away at a given point. Fig. 20-3rd and 4th provide two examples of this strategy.

Fig. 20-right shows the work cost calculated for these possible configurations. Note that the first configuration is the one which corresponds to the lowest cost. We also observed that the strategies that first compute the cost of regrouping one person only and then address the other people who have strayed are much more costly than other strategies.

Finally, in the third simulation, two robots guide a group of five people in 

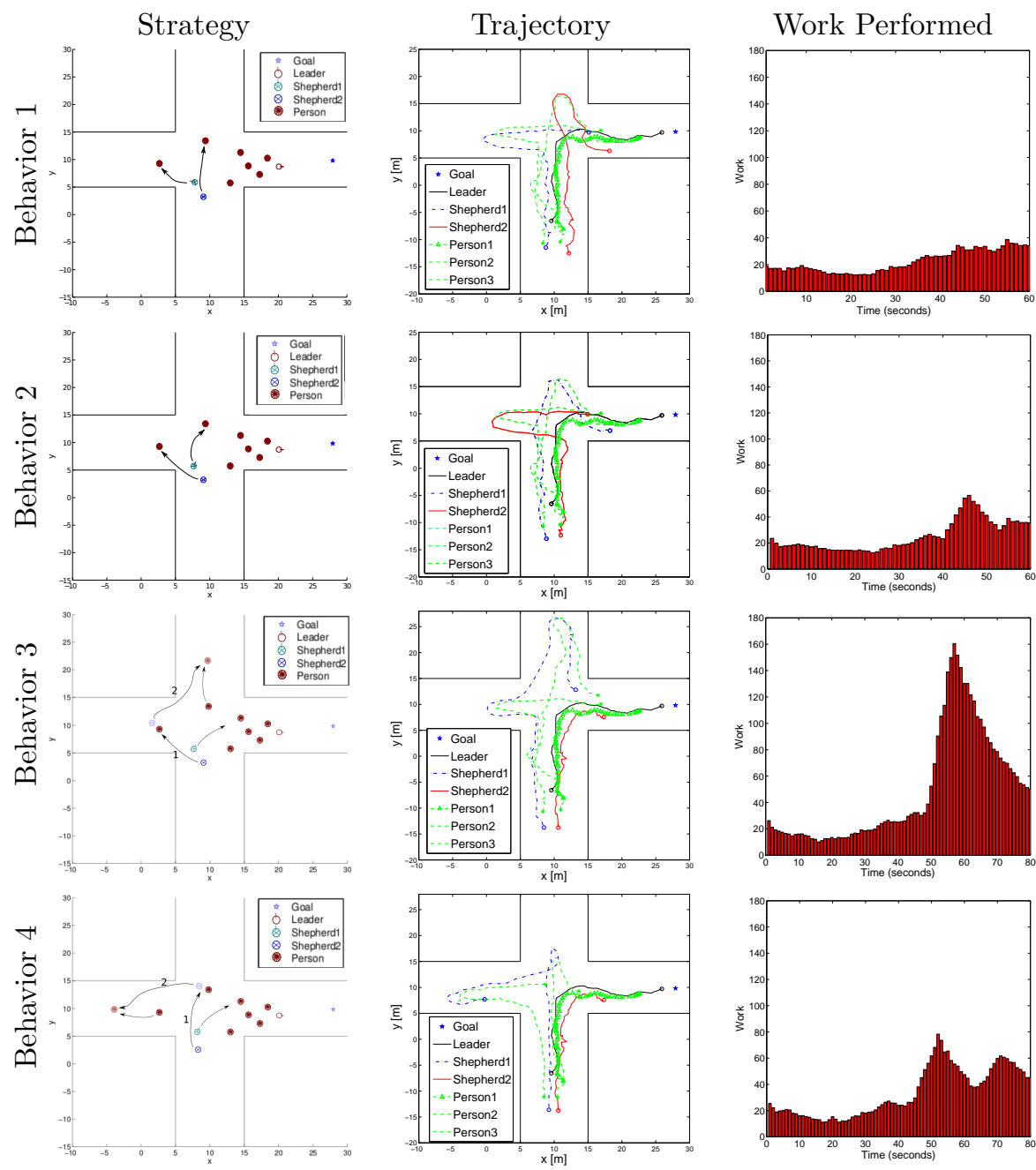

Figure 20: Simulations of Regrouping \#2: Left: Different strategies robots can follow. Center: Trajectories the group follows. Right: Work performed by the group.

a crossing area, see Fig. 21. The following are the possible configurations for regrouping people with two robots, one leader and one shepherd robot: (i) The shepherd robot takes care of regrouping people who have strayed following the right path Fig. 21-1st. (ii) The shepherd robot takes care of regrouping people who have strayed away following the left path, Fig. 21-2nd. (iii) The leader robot regroups the formation and the entire group moves toward the person who has strayed, Fig. 21-3rd. In (iv) and (v) the shepherd robot assumes the role of leader, left and right trajectories respectively, and the leader robot 

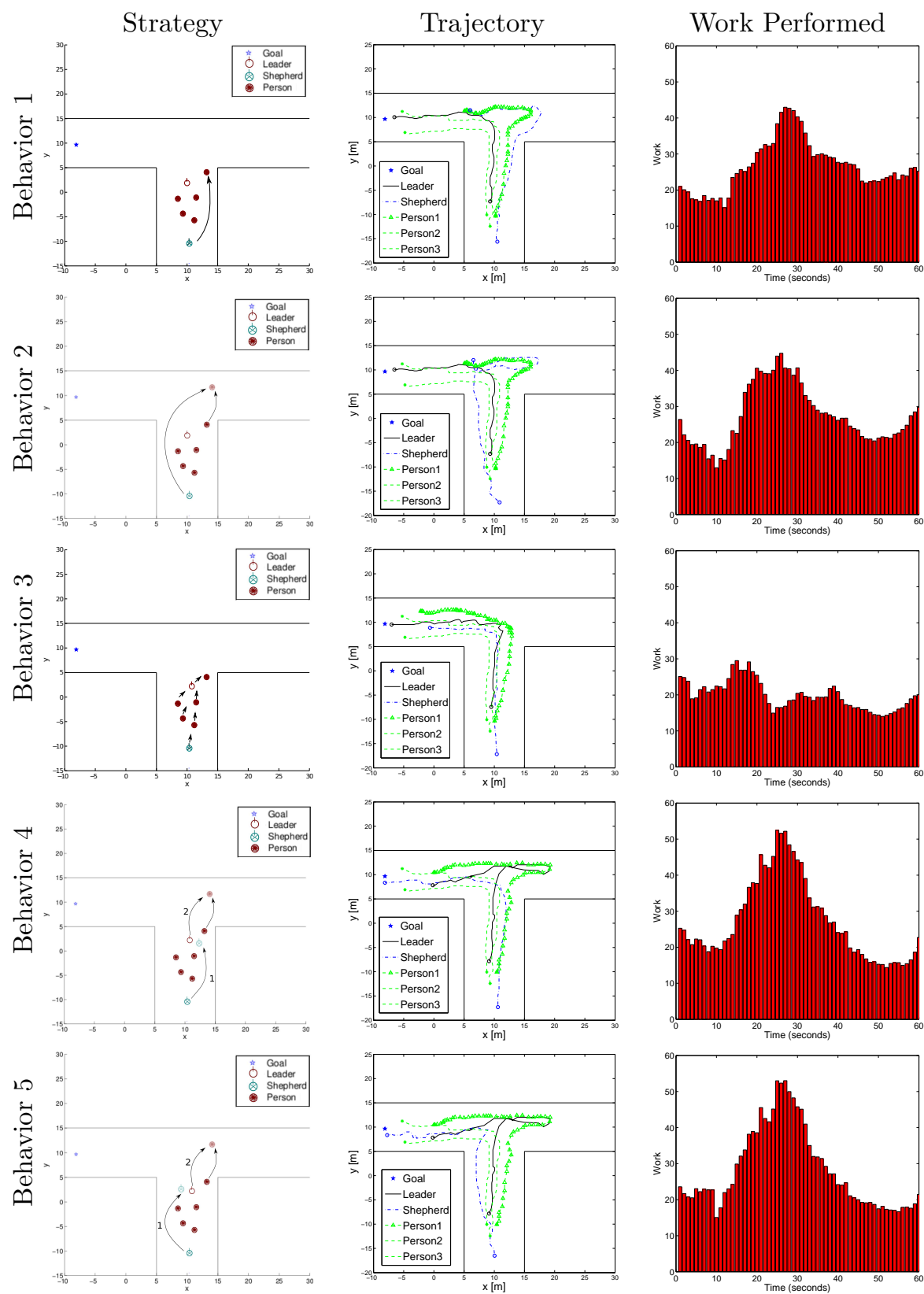

Figure 21: Simulation of the Regrouping \#3: Left: Different strategies that robots can follow. Center: Trajectories the group follows. Right: Work performed by the group. 
moves toward the person who is moving away. In these cases, we assume that the shepherd robot is able to explain that it has taken the role of the leader Fig. 21-4th and 5th. Fig. 21-right shows the work cost calculated for all possible configurations. Note that third configuration is the associated with the lowest cost.

In summary, we have presented different scenarios and several strategies with respect to the cost function in order to determine the ways in which robots can perform the given task cooperatively with the minimum work expended.

\section{Discussion, conclusions and future work}

To sum up, we shall discuss our conclusions, identify areas for further research and address the applicability of the model we have presented.

\subsection{Discussion}

We developed the Prediction and Anticipation Model, in order to guide people using multiple robots working cooperatively on a given task. We have also introduced the minimum robot motion energy criterion to assess the task assignment that might minimize the work required by people and robots during the guiding mission. Although the PAM model has been applied exclusively to people-guiding missions within the context of this paper, we believe it can be extended to other tasks that demand cooperation between robots and people, for example, robots gardening or robots cleaning in urban areas.

We have identified other works that use robots functioning cooperatively to guide people (Martinez Garcia et al., 2005), nevertheless, they do not tackle the problem of people straying from the formation. However, there are various methods for guiding people in museums using a single robot, as well as, guiding people inside a certain area using robot formation techniques or even guiding ducks within a controlled environment. Not all of these methods may be applied to the issue we mainly address in this work, since they do not allow people to stray from the group. Moreover, these techniques do not use the anticipation strategy to determine the best configuration of robots to carry out the mission.

We demonstrate the guiding mission using this model by conducting simulations instead of real-life experiments. The main reason for this decision is due to the complexity of conducting these experiments with the available technology. For example, the robots that exist nowadays do not move fast enough and do not navigate safely enough to follow people's random motions in an urban area. Nor may people's motions be completely and accurate detected when robots and persons are moving together in a group. These technological limitations will be overcome in the near future and we do not consider them to be significant challenges for the discipline in general. Furthermore, we have validated Helbing's forces using our robot Tibi and were able to calculate people's personal space when they interacted with Tibi. 
Because we have not demonstrated these trends in real-life experiments, we have had to make a series of assumptions on human motion behavior when people are guided by robots. Although one might believe that these assumptions are strong, we think they are valid wherever people cooperate with robots. Even if said assumptions are not partially or totally demonstrable in real-life scenarios, the minimum robot motion criterion can still be applied; we need only to include other criteria that consider human behavioral movement. These new criteria will need to be incorporated into the selection of the optimal robots configuration.

The proposed approach has some drawbacks, as described below. One drawback is time complexity of the optimal robot task assignment. As we mentioned earlier, this time complexity is $N_{R}$ !, meaning which, it grows factorially. We have not intended to look for techniques that reduce this time complexity, for example using heuristics, but this will be a task for a future work. Another drawback is the requirement of having to know the pose of all the people that move in the group. We think that we do not need all the poses, we only need the poses of the people that are in the border of the group, but this will be modified in the near future. At last, there is the drawback of the validation in real-life scenarios.

Lastly, we must note that the PAM model can be applied to real-life missions. Typical missions may include guiding tourists at a certain site; accompanying professional visitors in opened or close environments; service tasks such as cleaning or gardening in urban areas, etc.; and carrying out military missions. In all these tasks, people are gathered in small or large groups, and some of the participants may stray out of fatigue, boredom, injury, distraction, or for personal needs (such as finding a rest room). We have not considered situations in which the people in the group are in a state of panic or engaged in vandalism.

\subsection{Conclusions and future work}

This paper has presented a new model, called Prediction and Anticipation Model, which was developed to guide people using multiple robots that work cooperatively.

In contrast to other existing approaches, our method can handle realistic situations such as dealing with large environments with obstacles, or regrouping people who have left the group. For that reason, this work can be applied to certain specific real robot applications, for instance, guiding tourists, accompanying professional visitors or acting as a robot companion.

We have described the findings from various simulations conducted in open areas with obstacles and considered different people's behavioral movements. In each simulation, we were able to show that, by using the PAM model, the robots could act early enough and were able to prevent people from getting lost or straying from the group. We assert that these same results can be extended to urban areas with a large number of obstacles. Although we have only assessed the functionality of the PAM model through simulations, we have conducted real-life experiments to validate the Helbing's forces using our Tibi robot, and assessed the preferred dimensions of personal space, data which was 
then incorporated into our simulations.

Finally, we have identified three areas for further research: the first consists of adding into the particle filter sampling process the social force model, the second involves conducting real-life experiments in the Barcelona Robot Lab; and the third is to adjust the assumptions that were made in this work so that we might analyze new robot motion behaviors and strategies. We also hope to extend this model to other tasks where people and robots interact, such as, cleaning urban areas or gardening in urban spaces.

\section{References}

M.S. Arulampalam, S. Maskell, N. Gordon, T. Clapp, D. Sci, T. Organ, and S.A. Adelaide. A tutorial on particle filters for online nonlinear/nongaussianbayesian tracking. IEEE Transactions on Signal Processing, 50(2): 174-188, 2002.

R.W. Bohannon. Comfortable and maximum walking speed of adults aged 20-79 years: reference values and determinants. Age and ageing, 26(1):15, 1997.

W. Burgard, A. B. Cremers, D. Fox, D. Hahnel, G. Lakemeyer, D. Schulz, W. Steiner, and S. Thrun. The interactive museum tour-guide robot. Proc.of the Fifteenth National Conference on Artificial Intelligence, Madison, Wi, 1998.

J. Casper and R. R. Murphy. Human-robot interactions during the robotassisted urban search and rescue response at the world trade center. IEEE transactions on systems, man, and cybernetics, Part B., 33(3):367-385, 2003.

K. Dautenhahn. Robots as social actors: Aurora and the case of autism. In The Third International Cognitive Technology Conference, San Francisco, pages 359-374, 1999.

K. Dautenhahn, S. Woods, C. Kaouri, M. L. Walters, K. L. Koay, and I. Werry. What is a robot companion friend, assistant or butler? Proc. IEEE IROS, pages 1488-1493, 2005.

N. Davies, K. Cheverst, G. Blair, and K. Mitchell. Developing a context sensitive tourist guide. 1998.

D. Feil-Seifer and M.J. Mataric. Defining socially assistive robotics. In 9th International Conference on Rehabilitation Robotics., pages 465-468. IEEE, 2005 .

A. Garrell and A. Sanfeliu. Local optimization of cooperative robot movements for guiding and regrouping people in a guiding mission. In IEEE/RSJ International Conference on Intelligent Robots and Systems, pages 3294-3299, 2010a. 
A. Garrell and A. Sanfeliu. Model validation: Robot behavior in people guidance mission using DTM model and estimation of human motion behavior. In IEEE/RSJ International Conference on Intelligent Robots and Systems (IROS), pages 5836-5841, 2010b.

A. Garrell, A. Sanfeliu, and F. Moreno-Noguer. Discrete time motion model for guiding people in urban areas using multiple robots. In IEEE/RSJ International Conference on Intelligent Robots and Systems, pages 486-491. IEEE, 2009.

R.L. Goldstone, A. Jones, and M.E. Roberts. Group path formation. IEEE Transactions on Systems, Man and Cybernetics, Part A: Systems and Humans, 36(3):611-620, 2006.

N. B. Harrison. The language of shepherding. a pattern language for shepherds and sheep. Proc. 7th Pattern Languages of Programs Conference (PLoP), 1999.

K. Hayashi, D. Sakamoto, T. Kanda, M. Shiomi, S. Koizumi, H. Ishiguro, T. Ogasawara, and N. Hagita. Humanoid robots as a passive-social medium: a field experiment at a train station. In Proceedings of the ACM/IEEE international conference on Human-robot interaction, pages 137-144. ACM, 2007.

D. Helbing. A mathematical model for the behavior of pedestrians. Behavioral Science, 36(4), 1991.

D. Helbing and P. Molnar. Social force model for pedestrian dynamics. Physical Review E 51, pages 4282-4286, 1995.

D. Helbing and Molnar P. Self-organization phenomena in pedestrian crowds, in self-organization of complex structures: From individual to collective dynamics. F. Schweitzer (Ed.) (Gordon and Breach, London), pages 569-577, 1997.

G.S. Hornby, S. Takamura, J. Yokono, O. Hanagata, T. Yamamoto, and M. Fujita. Evolving robust gaits with aibo. In Robotics and Automation, 2000. Proceedings. ICRA'00. IEEE International Conference on, volume 3, pages 3040-3045. IEEE, 2000.

H. Ishiguro, T. Ono, M. Imai, T. Maeda, T. Kanda, and R. Nakatsu. Robovie: an interactive humanoid robot. Industrial robot: An international journal, 28 (6):498-504, 2001.

F. Kaplan. Talking aibo: First experimentation of verbal interactions with an autonomous four-legged robot. In Learning to behave: interacting agents CELE-TWENTE Workshop on Language Technology, pages 57-63. Citeseer, 2000.

K. Kawamura, R.T. Pack, M. Bishay, and M. Iskarous. Design philosophy for service robots. Robotics and Autonomous Systems, 18(1-2):109-116, 1996. ISSN 0921-8890. 
A. Konigs and D. Schulz. Fast visual people tracking using a feature-based people detector. In IEEE/RSJ International Conference on Intelligent Robots and Systems, pages 3614-3619. IEEE, 2011.

E. Kubinyi, Á. Miklósi, F. Kaplan, M. Gácsi, J. Topál, and V. Csányi. Social behaviour of dogs encountering aibo, an animal-like robot in a neutral and in a feeding situation. Behavioural processes, 65(3):231-239, 2004.

J. S. Liu and R. Chen. Sequential monte carlo methods for dynamic systems. Journal of the American Statistical Association, 93(443):1032-1044, 1998.

C. Marques and P. Lima. Multi-sensor navigation for soccer robots. RoboCup 2001: Robot Soccer World Cup V, pages 167-188, 2002.

E.A. Martinez Garcia, O. Akihisa, and S. Yuta. Crowding and guiding groups of humans by teams of mobile robots. IEEE Workshop on Advanced Robotics and its Social Impacts, pages 91-96, 2005.

G.F. Melson, P.H. Kahn Jr, A.M. Beck, B. Friedman, T. Roberts, and E. Garrett. Robots as dogs?: children's interactions with the robotic dog aibo and a live australian shepherd. In CHI'05 extended abstracts on Human factors in computing systems, pages 1649-1652. ACM, 2005.

A.C. Murtra, J.M.M. Tur, and A. Sanfeliu. Efficient active global localization for mobile robots operating in large and cooperative environments. In IEEE International Conference on Robotics and Automation, pages 2758-2763. IEEE, 2008.

I.R. Nourbakhsh, C. Kunz, and T. Willeke. The mobot museum robot installations: A five year experiment. In IEEE/RSJ International Conference on Intelligent Robots and Systems, volume 4, pages 3636-3641. IEEE, 2003.

A.K. Pandey and R. Alami. A step towards a sociable robot guide which monitors and adapts to the person's activities. In International Conference on Advanced Robotics., pages 1-8. IEEE, 2009.

J. Pransky. Social adjustments to a robotic future. www.robot.md/publications/sfra.html, pages 1-10, 2004.

S. Sabanovic, M.P. Michalowski, and R. Simmons. Robots in the wild: Observing human-robot social interaction outside the lab. In 9th IEEE International Workshop on Advanced Motion Control., pages 596-601. IEEE, 2006.

A. Sanfeliu and J. Andrade-Cetto. Ubiquitous networking robotics in urban settings. IEEE/RSJ International Conference on Intelligence Robots and Systems, Workshop on Network Robot Systems, 2006.

J.C. Scholtz. Human-Robot Interactions: Creating synergistic cyber Froces. In Multi-robot systems: from swarms to intelligent automata: proceedings from the NRL workshop on multi-robot systems, page 177, 2002. 
D. A. Shell and M. J. Matari. Insights toward robot-assisted evacuation. Advanced Robotics, 19(8):797-818, 2005.

M. Shiomi, T. Kanda, S. Koizumi, H. Ishiguro, and N. Hagita. Group attention control for communication robots with wizard of oz approach. In Proceedings of the ACM/IEEE international Conference on Human-Robot interaction, pages 121-128. ACM, 2007.

M. Shiomi, T. Kanda, D.F. Glas, S. Satake, H. Ishiguro, and N. Hagita. Field trial of networked social robots in a shopping mall. In IEEE/RSJ International Conference on Intelligent Robots and Systems, pages 2846-2853. IEEE, 2009.

M. Shiomi, T. Kanda, H. Ishiguro, and N. Hagita. A larger audience, please!: encouraging people to listen to a guide robot. In Proceeding of the 5th ACM/IEEE international conference on Human-robot interaction, pages 3138. ACM, 2010.

E.A. Sisbot, R. Alami, T. Simeon, K. Dautenhahn, M. Walters, and S. Woods. Navigation in the presence of humans. In 5th IEEE-RAS International Conference on Humanoid Robots., pages 181-188. IEEE, 2005.

C. Trevai, Y. Fukazawa, J. Ota, H. Yuasa, T. Arai, and H. Asama. Cooperative exploration of mobile robots using reaction-diffusion equation on a graph. IEEE International Conference on Robotics and Automation., 2, 2003.

E. Trulls, A. Corominas Murtra, J. Pérez-Ibarz, G. Ferrer, D. Vasquez, J.M. Mirats-Tur, and A. Sanfeliu. Autonomous navigation for mobile service robots in urban pedestrian environments. Journal of Field Robotics, 2011.

R. Vaughan. Experiments in Animal-interactive Robotics. PhD thesis, University of Oxford, 1998.

R.T. Vaughan, N. Sumpter, J. Henderson, A. Frost, and S. Cameron. Experiments in automatic flock control. J. Robot and Autonom. Sys., pages 191-209, 2000.

D.M. Wilkes, R.T. Pack, A. Alford, and K. Kawamura. Hudl, a design philosophy for socially intelligent service robots. In American Association for Artificial Intelligence, 1997.

S. Worchel, J. Cooper, G. R. Goethals, and J. M. Olson. Psicologia Social. Thomson Learning Ibero, 2002.

F. Zanlungo, T. Ikeda, and T. Kanda. Social force model with explicit collision prediction. EPL (Europhysics Letters), 93:68005, 2011. 